\title{
Drawing Planar Graphs with Few Geometric Primitives*
}

\author{
Gregor Hültenschmidt ${ }^{1}$, Philipp Kindermann², Wouter Meulemans ${ }^{3}$, and \\ André Schulz ${ }^{1}$ \\ ${ }^{1} L G$ Theoretische Informatik, FernUniversität in Hagen, Germany, \\ gregorhueltenschmidt@gmx.de, andre.schulz@fernuni-hagen.de \\ ${ }^{2}$ David R. Cheriton School of Computer Science, University of Waterloo, Canada, \\ pkinderm@uwaterloo.ca \\ ${ }^{3}$ TU Eindhoven, The Netherlands, w.meulemans@tue.nl
}

September 10, 2018

\begin{abstract}
We define the visual complexity of a plane graph drawing to be the number of basic geometric objects needed to represent all its edges. In particular, one object may represent multiple edges (e.g., one needs only one line segment to draw a path with an arbitrary number of edges). Let $n$ denote the number of vertices of a graph. We show that trees can be drawn with $3 n / 4$ straight-line segments on a polynomial grid, and with $n / 2$ straight-line segments on a quasi-polynomial grid. Further, we present an algorithm for drawing planar 3 -trees with $(8 n-17) / 3$ segments on an $O(n) \times O\left(n^{2}\right)$ grid. This algorithm can also be used with a small modification to draw maximal outerplanar graphs with $3 n / 2$ edges on an $O(n) \times O\left(n^{2}\right)$ grid. We also study the problem of drawing maximal planar graphs with circular arcs and provide an algorithm to draw such graphs using only $(5 n-11) / 3$ arcs. This is significantly smaller than the lower bound of $2 n$ for line segments for a nontrivial graph class.
\end{abstract}

\section{Introduction}

The complexity of a graph drawing can be assessed in a variety of ways: area, crossing number, bends, angular resolution, etc. All these measures have their justification, but in general it is impossible to optimize all of them in a single drawing. More recently, the visual complexity was suggested as another quality measure for drawings [24]. The visual complexity denotes the number of simple geometric entities used in the drawing.

Typically, we consider as entities either straight-line segments or circular arcs. To distinguish these two types of drawings, we call the former segment drawings and the latter arc drawings. The idea is that we can use, for example, a single segment to draw a path of collinear edges. The hope is that a drawing that consists of only a few geometric entities is easy to perceive. A recent user study [17] suggests that visual complexity may positively influence aesthetics, depending on the background of the observer, as long as

\footnotetext{
${ }^{*}$ A preliminary version of this work appeared in: Proc. 43rd Int. Workshop Graph-Theor. Concepts Comput. Sci. (WG'17) 14. The work of P. Kindermann and A. Schulz was supported by DFG grant SCHU 2458/4-1. The work of W. Meulemans was supported by Marie Skłodowska-Curie Action MSCAH2020-IF-2014 656741.
} 
Table 1: Upper bounds on the visual complexity. Here, $n$ is the number of vertices, $\vartheta$ the number of odd-degree vertices and $e$ the number of edges. Constant additions or subtractions have been omitted.

\begin{tabular}{|c|c|c|c|c|}
\hline \multirow{2}{*}{$\begin{array}{l}\text { Class } \\
\text { trees }\end{array}$} & \multicolumn{2}{|l|}{ Segments } & \multicolumn{2}{|c|}{ Arcs } \\
\hline & $\vartheta / 2$ & 10 & $\vartheta / 2$ & 10 \\
\hline maximal outerplanar & $n$ & 10 & $n$ & 10 \\
\hline 3 -trees & $2 n$ & 10 & $11 e / 18$ & 24 \\
\hline 3-connected planar & $5 n / 2$ & 10 & $2 e / 3$ & [24] \\
\hline cubic 3-connected planar & $n / 2$ & [15, 21] & $n / 2$ & [15, 21 \\
\hline triangulation & $7 n / 3$ & 11 & $5 n / 3$ & Thm. 5 \\
\hline 4-connected triangulation & $9 n / 4$ & 11 & $3 n / 2$ & Thm. 6 \\
\hline 4-connected planar & $9 n / 4$ & 11 & $9 n / 2-e$ & Thm. 8 \\
\hline planar & $16 n / 3-e$ & 11 & $14 n / 3-e$ & Thm. 7 \\
\hline
\end{tabular}

it does not introduce unnecessarily sharp corners. It is natural to ask for a drawing of a graph with the smallest visual complexity. Unfortunately, it is an NP-hard problem to determine the smallest number of segments necessary in a segment drawing [12. However, we can still expect to find algorithms, which guarantee bounds for certain graph classes.

Related work. For a number of graph classes, upper and lower bounds are known for segment drawings and arc drawings; the upper bounds are summarized in Table 1. However, these bounds (except for cubic 3-connected graphs) do not require the drawings to be on the grid. In his thesis, Mondal 20] gives an algorithm for triangulations with $n$ vertices using $8 n / 3-O(1)$ segments on a grid of size $2^{O(n \log n)}$ in general and of size $2^{O(n)}$ for triangulations of bounded degree. Even with this large grid, the algorithm uses substantially more segments than the best-known algorithm for triangulations without the grid requirement by Durocher and Mondal [11, which uses $7 n / 3-O(1)$ segments.

There are three trivial lower bounds for the number of segments required to draw any graph $G=(V, E)$ with $n$ vertices and $e$ edges:

(i) $\vartheta / 2$, where $\vartheta$ is the number of odd-degree vertices,

(ii) $\max _{v \in V}\lceil\operatorname{deg}(v) / 2\rceil$, and

(iii) $\lceil e /(n-1)\rceil$.

For triangulations and general planar graphs, a lower bound of $2 n-2$ and $5 n / 2-4$, respectively, is known [10]. Note that the trivial lower bounds are the same as for the slope number of graphs [26], that is, the minimum number of slopes required to draw all edges, and that the number of slopes of a drawing is upper bounded by the number of segments. Chaplick et al. 4, 5] consider a similar problem where all edges are to be covered by few lines (or planes); the difference to our problem is that collinear segments are counted only once in their model. In the same fashion, Kryven et al. 18 aim to cover all edges by few circles (or spheres).

Contributions. In this work, we present two types of results. In the first part (Sections 2, 3 and 4), we present algorithms for segment drawings on the grid with low visual complexity. This direction of research was posed as an open problem by Dujmović et al. [10, but only a few results exist; see Table 2. We present an algorithm that draws trees on an $O\left(n^{2}\right) \times O\left(n^{1.58}\right)$ grid using $3 n / 4$ straight-line segments. For comparison, the drawings of Schulz [24] need also $3 n / 4$ arcs on a smaller $O(n) \times O\left(n^{1.58}\right)$ grid, but use the more complex circular arcs instead. Our segment drawing algorithm for trees can be mod- 
Table 2: Same as in Table1 but for grid drawings.

\begin{tabular}{|c|c|c|c|c|}
\hline Class & Type & Compl. & Grid & Ref. \\
\hline trees & $\operatorname{arcs}$ & $3 n / 4$ & $O(n) \times O\left(n^{1.58}\right)$ & [24] \\
\hline trees & segments & $3 n / 4$ & $\mathbf{O}\left(\mathbf{n}^{2}\right) \times \mathbf{O}\left(\mathbf{n}^{1.58}\right)$ & Thm. 1 \\
\hline trees & segments & $\vartheta / \mathbf{2}$ & quasi-polynom. & Thm. $\overline{\overline{2}}$ \\
\hline cubic planar 3-conn. & segments & $n / 2$ & $O(n) \times O(n)$ & [15, 21] \\
\hline maximal outerplanar & segments & $3 n / 2$ & $\mathbf{O}(\mathbf{n}) \times \mathbf{O}\left(\mathbf{n}^{2}\right)$ & Thm. 4 \\
\hline triangulation & segments & $8 n / 3$ & $O\left((3.63 n)^{4 n / 3}\right)$ & [20] \\
\hline planar 3-tree & segments & $8 n / 3$ & $\mathbf{O}(\mathbf{n}) \times \mathbf{O}\left(\mathbf{n}^{2}\right)$ & Thm. 3 \\
\hline
\end{tabular}

ified to generate drawings with an optimal number of $\vartheta / 2$ segments on a quasi-polynomial grid. We also present algorithms to compute segment drawings of planar 3-trees and maximal outerplanar graphs, both on an $O(n) \times O\left(n^{2}\right)$ grid. In the case of planar 3-trees, the algorithm needs at most $(8 n-17) / 3$ segments, and in the case of maximum outerplanar graphs the algorithm needs at most $3 n / 2$ segments.

Finally, in sections 5 and 6 , we study arc drawings of triangulations and general planar graphs. In particular, we prove that $(5 n-11) / 3$ arcs are sufficient to draw any triangulation with $n$ vertices. We highlight that this bound is significantly smaller than the $2 n-2$ lower bound known for segment drawings [10] and the so far best-known $2 e / 3=2 n$ upper bound for circular arc drawings [24]. A straightforward extension shows that $(14 n-3 e-29) / 3$ arcs are sufficient for general planar graphs with $e$ edges.

Preliminaries. Given a triangulated planar graph $G=(V, E)$ on $n$ vertices, a canonical order $\sigma=\left(v_{1}, \ldots, v_{n}\right)$ is an ordering of the vertices in $V$ such that, for $3 \leq k \leq n$, (i) the subgraph $G_{k}$ of $G$ induced by $v_{1}, \ldots, v_{k}$ is biconnected, (ii) the outer face of $G_{k}$ consists of the edge $\left(v_{2}, v_{1}\right)$ and a path $C_{k}$, called contour, that contains $v_{k}$, and (iii) the neighbors of $v_{k}$ in $G_{k-1}$ form a subpath of $C_{k-1}$ [8, 9]. A canonical order can be constructed in reverse order by repeatedly removing a vertex without an incident chord from the outer face.

Most of our algorithms make ample use of Schnyder realizers 23. Assume we selected a face as the outer face with vertices $v_{1}, v_{2}$ and $v_{n}$. We decompose the interior edges into three trees: $T_{1}, T_{2}$, and $T_{n}$ rooted at $v_{1}, v_{2}$, and $v_{n}$, respectively. The edges of the trees are oriented to their roots. For $k \in\{1,2, n\}$, we call each edge in $T_{k}$ a $k$-edge and the parent of a vertex in $T_{k}$ its $k$-parent. In the figures of this paper, we will draw 1-edges red, 2-edges blue, and $n$-edges green. The decomposition is a Schnyder realizer if at every interior vertex the edges are cyclically ordered as: outgoing 1-edge, incoming $n$-edges, outgoing 2 -edge, incoming 1-edges, outgoing $n$-edge, incoming 2-edges. The trees of a Schnyder realizer are also called canonical ordering trees, as each describes a canonical order on the vertices of $G$ by a (counter-)clockwise pre-order traversal [7]. There is a unique minimal realizer such that any interior cycle in the union of the three trees is oriented clockwise [3]; this realizer can be computed in linear time [3, 23]. The number of such cycles is denoted by $\Delta_{0}$ and is upper bounded by $\lfloor(n-1) / 2\rfloor[27$. Bonichon et al. [1] prove that the total number of leaves in a minimal realizer is at most $2 n-5-\Delta_{0}$.

\section{Trees with segments on the grid}

Let $T=(V, E)$ be an undirected tree. Our algorithm follows the basic idea of the circular arc drawing algorithm by Schulz [24]. We make use of the heavy path decomposition [25] of 


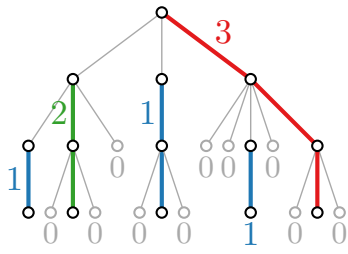

(a) A heavy path decomposition and the depths.

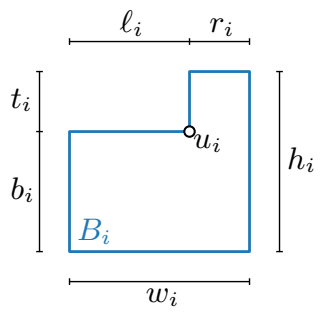

(b) The heavy path box $B_{i}$ with top node $u_{i}$.

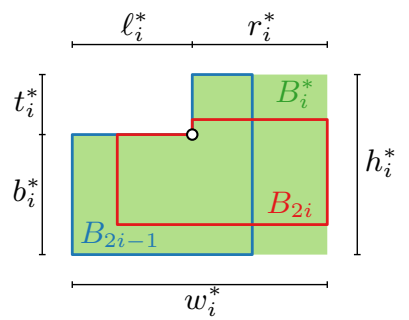

(c) The merged box $B_{i}^{*}$ for $B_{2 i-1}$ and $B_{2 i}$.

Figure 1: The heavy-path boxes and merged boxes with their respective lengths.

trees, which is defined as follows. First, root the tree at some vertex $r$ and direct all edges away from the root. Then, for each non-leaf $u$, compute the size of each subtree rooted in one of its children. Let $v$ be the child of $u$ with the largest subtree (one of them in case of a tie). Then, $(u, v)$ is called a heavy edge and all other outgoing edges of $u$ are called light edges. The maximal connected components of heavy edges form the heavy paths of the decomposition.

We call the vertex of a heavy path closest to the root its top node and the subtree rooted in the top node a heavy path subtree. We define the depth of a heavy path (subtree) as follows. We treat each leaf that is not incident to a heavy edge as a heavy path of depth 0 . The depth of each other heavy path $P$ is by 1 larger than the maximum depth of all heavy paths that are connected from $P$ by an outgoing light edge. Heavy path subtrees of common depth are disjoint.

Boxes. We order the heavy paths nondecreasingly by their depth and then draw their subtrees in this order. Each heavy path subtree is placed completely inside an L-shaped box (heavy path box) with its top node placed at the reflex angle; see Fig. $1 \mathrm{~b}$ for an illustration of a heavy path box $B_{i}$ with top node $u_{i}$, width $w_{i}=\ell_{i}+r_{i}$, and height $h_{i}=$ $t_{i}+b_{i}$. We require that

(i) heavy-path boxes of common depth are disjoint,

(ii) $u_{i}$ is the only vertex on the boundary of $B_{i}$, and

(iii) $b_{i} \geq t_{i}$.

Note that the boxes will be mirrored horizontally and/or vertically in some steps of the algorithm. We assign to each heavy path subtree of depth 0 a heavy path box $B_{i}$ with $\ell_{i}=r_{i}=t_{i}=b_{i}=1$.

Drawing. Since a heavy path subtree of depth 0 consists of exactly one vertex that is placed in a box as described above, we have already drawn all heavy path subtrees of depth 0 . We will inductively draw the heavy path subtrees by their depth. Assume that we have already drawn each heavy path subtree of depth $d \geq 0$. In the $(d+1)$-th step of our algorithm, we will draw all heavy path subtrees of depth $d+1$. Let $\left\langle v_{1}, \ldots, v_{m}\right\rangle$ be a heavy path of depth $d+1$. We proceed as follows; see Fig. 2a for an illustration. The last vertex on a heavy path has to be a leaf, so $v_{m}$ is a leaf.

If the outdegree of $v_{m-1}$ is odd, then we place the vertices $v_{1}, \ldots, v_{m}$ on a vertical line; otherwise, we place only the vertices $v_{1}, \ldots, v_{m-1}$ on a vertical line and treat $v_{m}$ as a heavy path subtree of depth 0 that is connected to $v_{m-1}$.

For $1 \leq h \leq m-1$, all heavy-path boxes adjacent to $v_{h}$ will be drawn either in a rectangle on the left side of the edge $\left(v_{h}, v_{h+1}\right)$ or in a rectangle on the right side of the edge $\left(v_{h-1}, v_{h}\right)$ (a rectangle that has $v_{1}$ as its bottom left corner for $h=1$ ). 


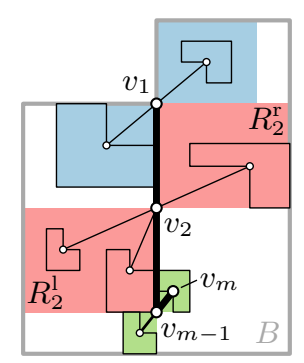

(a)

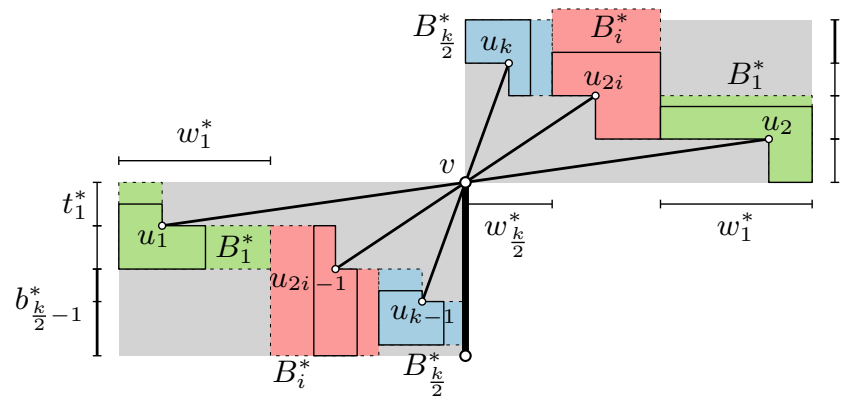

(b)

Figure 2: (a) Placement of a heavy path, its box $B$, and areas for the adjacent heavy path boxes. (b) Placement of the heavy-path boxes adjacent to $v$.

We now describe how to place the heavy-path boxes $B_{1}, \ldots, B_{k}$ with top node $u_{1}, \ldots, u_{k}$, respectively, adjacent to some vertex $v$ on a heavy path into the rectangles described above; see Fig. $2 \mathrm{~b}$ for an illustration.

First, assume that $k$ is even. Then, for $1 \leq i \leq k / 2$, we order the boxes such that $b_{2 i} \leq b_{2 i-1}$. We place the box $B_{2 i-1}$ in the lower left rectangle and box $B_{2 i}$ in the upper right rectangle in such a way that the edges $\left(v, u_{2 i-1}\right)$ and $\left(v, u_{2 i}\right)$ can be drawn with a single segment. To this end, we construct a merged box $B_{i}^{*}$ as depicted in Fig. $1 \mathrm{c}$ with $\ell_{i}^{*}=\max \left\{\ell_{2 i-1}, \ell_{2 i}\right\}, r_{i}^{*}=\max \left\{r_{2 i-1}, r_{2 i}\right\}$, and $w_{i}^{*}=\ell_{i}^{*}+r_{i}^{*}$; the heights are defined analogously. The merged boxes will help us reduce the number of segments.

We mirror all merged boxes horizontally and place them in the lower left rectangle (of width $\left.\sum_{i=1}^{k / 2} w_{i}^{*}\right)$ as follows. We place $B_{1}^{*}$ in the top left corner of the rectangle. For $2 \leq$ $j \leq k / 2$, we place $B_{j}^{*}$ directly to the right of $B_{j-1}^{*}$ such that its top border lies exactly $t_{j-1}^{*}$ rows below the top border of $B_{j-1}^{*}$. Symmetrically, we place the merged boxes (vertically mirrored) in the upper right rectangle.

Finally, we place each box $B_{2 i-1}$ (horizontally mirrored) in the lower left copy of $B_{i}^{*}$ such that their inner concave angles coincide, and we place each box $B_{2 i}$ (vertically mirrored) in the upper right copy of $B_{i}^{*}$ such that their inner concave angles coincide.

If $k$ is odd, then we simply add a dummy box $B_{k+1}=B_{k}$ that we remove afterwards.

The box of the heavy path subtree $\left\langle v_{1}, \ldots, v_{m}\right\rangle$ is the smallest box that contains $v_{1}, \ldots, v_{m}$ as well as the rectangles next to these vertices (see Fig 2a). We proceed the same way for every other heavy path subtree of depth $d+1$; thus, we have obtained a drawing and its box for every heavy path subtree of depth $d+1$.

Analysis. We will now calculate the width and the height of this construction.

Lemma 1. Any heavy path subtree of depth $d$ that contains $n^{\prime}$ vertices is drawn in a heavy-path box $B$ with width $w=\ell+r \leq 2^{d+1} \cdot n^{\prime}$ and height $h=b+t \leq 2 \cdot(3 / 2)^{d} \cdot n^{\prime}$ and $b \geq t$, where $\ell, r, b, t$ are defined as in Fig. $1 b$.

Proof. We prove the lemma by induction over $d$.

Recall that we place the depth-0 heavy paths in a box of width $2=2^{1} \cdot 1$ and height $2=2 \cdot(3 / 2)^{0} \cdot 1$, so the bounds hold for $d=0$. Assume that the bound holds for all heavy path subtrees of depth $d \geq 0$.

Let $\left\langle v_{1}, \ldots, v_{m}\right\rangle$ be a heavy path of depth $d+1$. Consider the drawing of the heavy path subtree rooted in $v_{1}$ as described above. Let $R_{j}^{l}$ be the left rectangle and let $R_{j}^{\mathrm{r}}$ be the right rectangle that contain all heavy-path boxes adjacent to $v_{j}$ for $1 \leq j \leq m-1$; see Fig. 2a. We denote by $w_{j}^{1}$ and $h_{j}^{1}$ the width and the height of $R_{j}^{\mathrm{l}}$, respectively, and by $w_{j}^{\mathrm{r}}$ 
and $h_{j}^{\mathrm{r}}$ the width and the height of $R_{j}^{\mathrm{r}}$, respectively. Since $v_{m}$ is a leaf, we have $w_{m}^{\mathrm{l}}=$ $w_{m}^{\mathrm{r}}=h_{m}^{\mathrm{l}}=h_{m}^{\mathrm{r}}=1$.

We will first analyze the width and height of these rectangles for a fixed vertex $v_{j}$, $1 \leq j \leq m-1$. Let $u_{1}^{j}, \ldots, u_{k_{j}}^{j}$ be the children of $v_{j}$ and denote the number of nodes in the heavy path subtrees rooted in them by $n_{1}^{j}, \ldots, n_{k_{j}}^{j}$. Let $n_{j}^{\prime}=\sum_{i=1}^{k_{j}} n_{i}^{j}$; note that $\sum_{j=1}^{m} n_{j}^{\prime}=n^{\prime}-m$. By induction, we have $w_{i}^{j} \leq 2^{d+1} \cdot n_{i}^{j}$ for $1 \leq i \leq k_{j}$ and $h_{i}^{j}=b_{i}^{j}+t_{i}^{j} \leq$ $2 \cdot(3 / 2)^{d} \cdot n_{i}^{j}$.

For the width of the rectangles, we obtain

$$
\begin{aligned}
w_{j}^{1}=w_{j}^{\mathrm{r}} & \leq 1+\sum_{i=1}^{k_{j} / 2} w_{i}^{j *}=1+\sum_{i=1}^{k_{j} / 2} \max \left\{w_{2 i-1}^{j}, w_{2 i}^{j}\right\} \leq 1+\sum_{i=1}^{k_{j}} w_{i}^{j} \\
& \leq 1+\sum_{i=1}^{k_{j}}\left(2^{d+1} \cdot n_{i}^{j}\right)=1+2^{d+1} \sum_{i=1}^{k_{j}} n_{i}^{j} \\
& \leq 2^{d+1}\left(1+n_{j}^{\prime}\right) .
\end{aligned}
$$

The height of each rectangle in the construction is at least $2 \sum_{i=1}^{k_{j} / 2} t_{i}^{j *}$, but we have to add a bit more for the bottom parts of the boxes; in the worst case, this is $\max _{1 \leq \lambda \leq k_{j} / 2} b_{2 \lambda-1}^{j}$ in $R_{j}^{\mathrm{l}}$ and $\max _{1 \leq \mu \leq k_{j} / 2} b_{2 \mu}$ in $R_{j}^{\mathrm{r}}$. Since our constructions ensures that $b_{i}^{j} \geq t_{i}^{j}$, we have

$$
\begin{aligned}
h_{j}^{1}+h_{j}^{\mathrm{r}} & \leq 1+2 \sum_{i=1}^{k_{j} / 2} t_{i}^{j *}+\max _{1 \leq \lambda \leq k_{j} / 2} b_{2 \lambda-1}^{j}+\max _{1 \leq \mu \leq k_{j} / 2} b_{2 \sigma}^{j} \\
& \leq 1+2 \sum_{i=1}^{k_{j}} t_{i}^{j}+\sum_{i=1}^{k_{j}} b_{i}^{j} \leq 1+x \frac{3}{2} \sum_{i=1}^{k_{j}} h_{i}^{j} \\
& \leq 1+\frac{3}{2} \sum_{i=1}^{k_{j}}\left(2 \cdot\left(\frac{3}{2}\right)^{d} \cdot n_{i}^{j}\right)=1+2 \cdot\left(\frac{3}{2}\right)^{d+1} \sum_{i=1}^{k_{j}} n_{i}^{j} \\
& \leq 2 \cdot\left(\frac{3}{2}\right)^{d+1}\left(n_{j}^{\prime}+1\right) .
\end{aligned}
$$

We will now analyze the width and height of the heavy path box of the heavy path subtree rooted in $v_{1}$. For the width, we have $w=\ell+r$ with $\ell \leq \max _{1 \leq j \leq m} w_{j}^{1}$ and $r \leq \max _{1 \leq j \leq m} w_{j}^{\mathrm{r}}=\ell$. Hence, we obtain

$$
\begin{aligned}
w & =2 \ell=2 \max _{1 \leq j \leq m} w_{j}^{1} \leq 2 \sum_{j=1}^{m} w_{j}^{1} \\
& \leq 2+2 \sum_{j=1}^{m-1}\left(2^{d+1}\left(n_{j}^{\prime}+1\right)\right)=2+2^{d+2} \sum_{j=1}^{m-1}\left(n_{j}^{\prime}+1\right) \\
& =2+2^{d+2} \cdot\left(n^{\prime}-1\right) \leq 2^{d+2} \cdot n^{\prime} .
\end{aligned}
$$

For the height of the heavy path box, notice that the vertical distance between two vertices $v_{j-1}$ and $v_{j}, 2 \leq j \leq m$, is at most $\max \left\{h_{j-1}^{1}, h_{j}^{\mathrm{r}}\right\}$, while the vertical space below between $v_{m}$ is at most $h_{m}^{1}=1$. Hence, we have 


$$
\begin{aligned}
h & =t+b \leq h_{1}^{\mathrm{r}}+\sum_{j=2}^{m} \max \left\{h_{j-1}^{\mathrm{l}}, h_{j}^{\mathrm{r}}\right\}+h_{m}^{\mathrm{l}} \\
& \leq \sum_{j=1}^{m}\left(h_{j}^{1}+h_{j}^{\mathrm{r}}\right)=2+\sum_{j=1}^{m-1}\left(h_{j}^{1}+h_{j}^{\mathrm{r}}\right) \\
& \leq 2+\sum_{j=1}^{m-1}\left(2 \cdot\left(\frac{3}{2}\right)^{d+1}\left(n_{j}^{\prime}+1\right)\right)=2+2 \cdot\left(\frac{3}{2}\right)^{d+1}\left(n^{\prime}-1\right) \\
& \leq 2 \cdot\left(\frac{3}{2}\right)^{d+1} n^{\prime} .
\end{aligned}
$$

Since all heavy path trees of common depth are disjoint, the heavy-path boxes of common depth are as well. Further, we place only the top vertex of a heavy path on the boundary of its box. Finally, since we order the boxes such that $b_{2 i-1}^{j} \geq b_{2 i}^{j}$ for each $i$ and $j$, we have $h_{j}^{1} \geq h_{j}^{\mathrm{r}}$ for each $j$ and thus $b \geq t$.

Due to the properties of a heavy path decomposition, the maximum depth of any heavy path is $\lceil\log n\rceil$. Thus, by Lemma 1 , the whole tree is drawn in a box of width $2 \cdot 2^{\lceil\log n\rceil} n=O\left(n^{2}\right)$ and height $2 \cdot(3 / 2)^{\lceil\log n\rceil} n=O\left(n^{1+\log 3 / 2}\right) \subseteq O\left(n^{1.58}\right)$.

By using the same heavy path decomposition as the algorithm of Schulz [24] (that is, we choose the same heavy edge in case of a tie), we have that our algorithm draws a path in $T$ with one segment if and only if the algorithm of Schulz draws this path with one circular arc. Hence, following his analysis, our drawing uses at most $\lceil 3 e / 4\rceil=\lceil 3(n-1) / 4\rceil$ segments.

Theorem 1. Every tree admits a straight-line drawing that uses at most $\lceil 3 e / 4\rceil$ segments on an $O\left(n^{2}\right) \times O\left(n^{1.58}\right)$ grid. This drawing can be computed in $O(n)$ time.

Proof. The existence of the drawing is already argued above; what remains is to prove the time bound. By traversing the tree bottom-up, we can calculate the heavy path decomposition in $O(n)$ time. Then, we sort the heavy paths by their depth in $O(n)$ time using, e.g., counting sort since the depth is integer and bound by $O(\log n)$.

When drawing a heavy path of level $d$, we only have to place the adjacent heavy-path boxes of level $d-1$ around the vertices of the heavy path, which gives us their coordinates and the corresponding heavy path box. The number of these placement steps is equal to the number of light edges in the graph, which is $O(n)$.

We finish this section with an adjustment of our algorithm to get a grid drawing with the best possible number of straight-line segments. Observe that there is only one situation in which the previous algorithm uses more segments than necessary, that is the top node of each heavy path. The heavy path is always drawn vertically, while the incoming light edge of the top node will be drawn with a different slope; aligning the two slopes would reduce the number of segments. This suboptimality can be "repaired" by tilting the heavy path as sketched in Fig. 3. Note that the incident subtrees with smaller depth will only be translated. However, when drawing a heavy path box, we do not know yet with which slope the incoming light edge will be drawn.

Our adjustment works as follows. We first draw each heavy path vertically and then change its slope when placing the corresponding heavy path box into a rectangle next to the parent of its top node. Assume that we have already drawn each heavy path subtree 

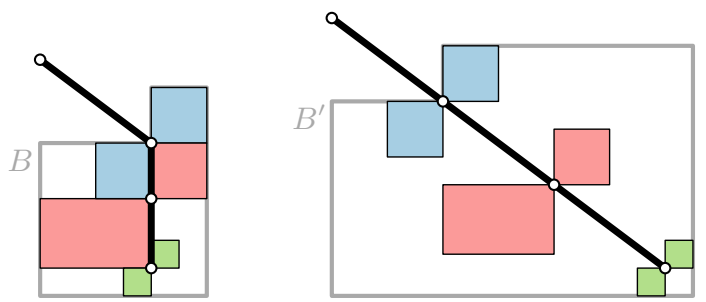

Figure 3: Further improvement on the visual complexity via increasing the size of the boxes.

of depth $d$ such that their heavy path is drawn vertically. Let $v$ be some vertex on a heavy path of depth $d+1$ and let $B_{1}, \ldots, B_{k}$ be the heavy-path boxes with top node $u_{1}, \ldots, u_{k}$, respectively, adjacent to $v$.

Note that the tilting of a heavy path only increases the right and the bottom length of its heavy-path box; in particular, if $B_{i}$ is a heavy path box with width $w_{i}=\ell_{i}+r_{i}$ and height $h_{i}=t_{i}+b_{i}$ and $B_{i}^{\prime}$ is the tilted heavy path box with width $w_{i}^{\prime}=\ell_{i}^{\prime}+r_{i}^{\prime}$ and height $h_{i}^{\prime}=t_{i}^{\prime}+b_{i}^{\prime}$, then we have $\ell_{i}^{\prime}=\ell_{i}, r_{i}^{\prime}>r_{i}, t_{i}^{\prime}=t_{i}$, and $b_{i}^{\prime}>b_{i}$. The $y$-coordinate of the vertices $u_{1}, \ldots, u_{k}$ after the placement depends only of the values $t_{1} \ldots, t_{k}$, so they will stay the same after the tilting procedure. Hence, we can determine the $y$-coordinates by running the previous drawing algorithm and then place the boxes in reverse order ("inside-out" instead of top-down). Then, the $x$-coordinate of each vertex $u_{i}$ depends only on the width of the already placed boxes $u_{i+1}, \ldots, u_{k}$ and of $r_{i}=r_{i}^{\prime}$, so we know exactly by which slope we have to tilt the box $B_{i}$. Finally, to tilt the box $B_{i}$, we change the vector of each edge $e$ on its heavy path to be an integer multiple of the vector of the edge from $v$ to $u_{i}$ such that the vertical length of $e$ is not decreased. This keeps the rectangles that contain the adjacent lower-level heavy-path boxes disjoint.

Unfortunately, this procedure increases the area of the drawing. By tilting the heavypath boxes, we have to blow up their size. We are left with scaling in each "round" by a polynomial factor. Since there are only $\log n$ rounds, we obtain a drawing on a quasi-polynomial grid. However, an implementation of the algorithm shows that some simple heuristics can already substantially reduce the drawing area, which gives hope that drawings on a polynomial grid exist for all trees; see Fig. 4 .

Theorem 2. Every tree admits a straight-line drawing with the smallest number of straightline segments on a quasi-polynomial grid. This drawing can be computed in $O(n)$ time.

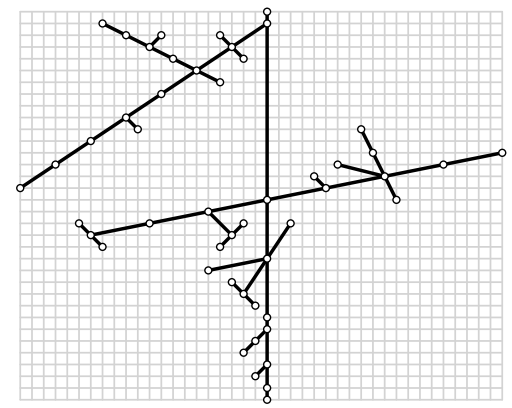

(a) The drawing produced by our algorithm.

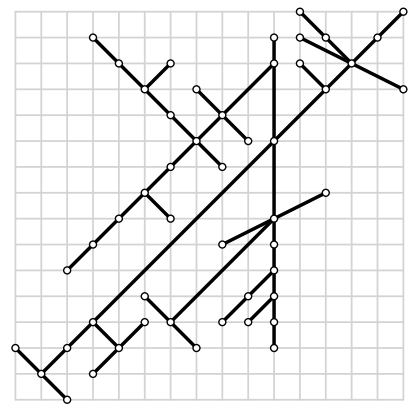

(b) Heuristic improvement on the drawing area.

Figure 4: Drawings of a tree with 50 vertices with minimum number of segments. 
Proof. The existence of the drawing is argued above. The time bound is the same as in Theorem 1 as the only new step is tilting the heavy-path boxes, which changes the slope of each heavy edge at most once.

\section{Planar 3-trees with few segments on the grid}

In this section, we show how to construct drawings of planar 3-trees with few segments on the grid. We begin by introducing some notation. A 3-tree is a maximal graph of treewidth $k$, that is, no edges can be added without increasing the treewidth. Each planar 3-tree can be produced from the complete graph $K_{4}$ by repeatedly adding a vertex into a triangular face and connecting it to all three vertices incident to this face. This operation is also known as stacking. Any planar 3-tree admits exactly one Schnyder realizer, and it is cycle-free [3], that is, directing all edges according to $T_{1}, T_{2}$, and $T_{n}$ gives a directed acyclic graph.

Let $\mathcal{T}$ be a planar 3 -tree. Let $T_{1}, T_{2}$, and $T_{n}$ rooted at $v_{1}, v_{2}$, and $v_{n}$, respectively, be the canonical ordering trees of the unique Schnyder realizer of $\mathcal{T}$. Recall that each canonical ordering tree describes a canonical order on the vertices of $T$ by a (counter-)clockwise pre-order traversal [7]. Without loss of generality, let $T_{1}$ be the canonical ordering tree having the fewest leaves, and let $\sigma=\left(v_{1}, v_{2}, \ldots, v_{n}\right)$ be the canonical order induced by a clockwise pre-order walk of $T_{2}$; see Fig. 5 . The fact that we choose the canonical order induced by $T_{2}$ instead of $T_{1}$ may seem counter-intuitive, but we will require a specific property of the canonical order that is ensured only by this choice later. The following lemma holds for any $v_{k}, 4 \leq k \leq n$.

Lemma 2. Let $C_{k-1}=\left(w_{1}, \ldots, w_{r}\right)$ be the contour of $G_{k-1}$, let $w_{p}$ be the 1-parent of $v_{k}$, and let $w_{q}$ be the 2-parent of $v_{k}$. Then,

(a) either $\left(w_{q}, w_{p}\right) \in T_{1}$ or $\left(w_{p}, w_{q}\right) \in T_{2}$

(b) the subgraph inside the triangle $\left(w_{p}, w_{q}, v_{k}\right)$ is a planar 3-tree

(c) if $q>p+1$, then $w_{p+1}, \ldots, w_{q-1}$ lie inside the triangle $\left(w_{p}, w_{q}, v_{k}\right),\left(w_{p+1}, w_{p}\right) \in T_{1}$, and $\left(w_{q-1}, w_{q}\right) \in T_{2}$.

Proof. Proof for (a): For $q=p+1$ the statement holds trivially. So we assume that there

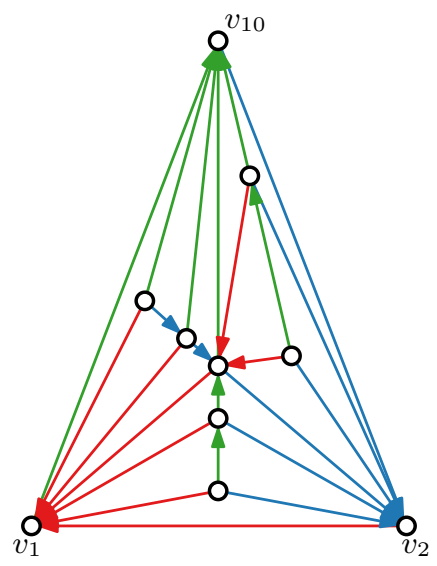

(a)

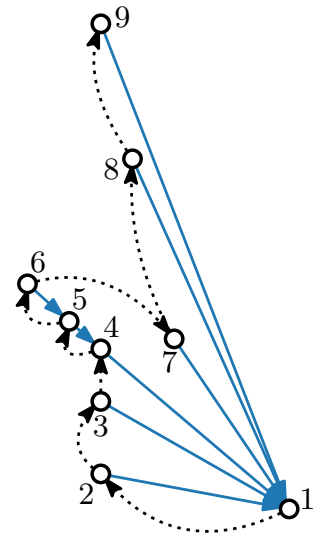

(b)

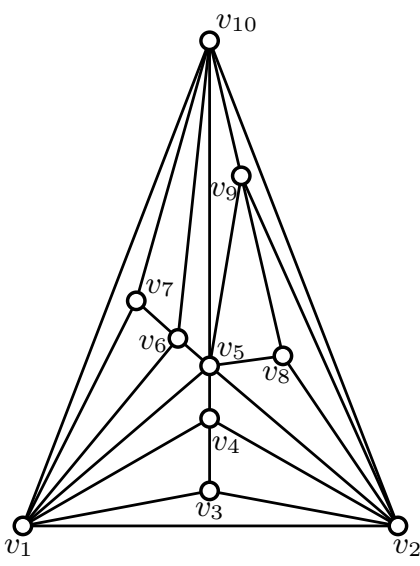

(c)

Figure 5: Obtaining a canonical order from a clockwise pre-order traversal of a canonical ordering tree. (a) The canonical ordering trees of a planar 3-tree; (b) the clockwise preorder traversal on $T_{2}$; (c) the canonical order induced by this traversal. 


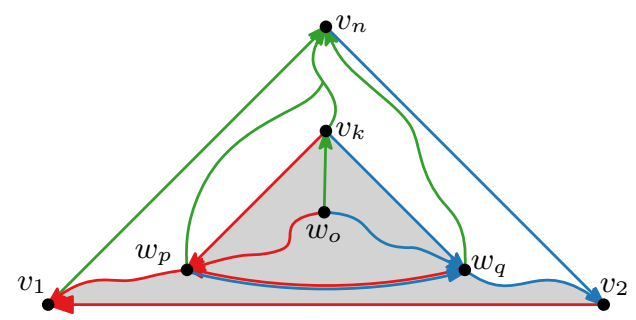

Figure 6: Proof that the edge $\left(w_{p}, w_{q}\right)$ exists.

exists a vertex $w_{o}$ on $C_{k}$ between $w_{p}$ and $w_{q}$. Let $X=\left\{v_{n}\right\}$ and $Y=\left\{w_{o}\right\}$. Obviously, the contour $C_{k}$ is an $X-Y$-separator; see Fig. 6. Let $Z$ be a minimal $X-Y$-separator that contains only vertices from $C_{k}$. By the properties of the Schnyder realizer, each incoming $n$-edge of a vertex on $C_{k}$ comes from a vertex below the contour. Hence, there is a path from each vertex on $C_{k}$ to $v_{n}$ in $T_{n}$ that does not revisit $C_{k}$. Further, the paths $\left(w_{o}, v_{k}\right)$, $\left(w_{o}, w_{o-1}, \ldots, w_{p}\right)$, and $\left(w_{o}, w_{o+1}, \ldots, w_{q}\right)$ connect $w_{o}$ to each of $v_{k}, w_{p}$ and $w_{q}$ without traversing any other vertex from $C_{k}$. Hence, $Z$ has to contain $v_{k}, w_{p}$, and $w_{q}$; otherwise, a $v_{n}-w_{o}$-path would remain after deleting $Z$. Rose 22 has shown that every minimal $X-Y$-separator in a $k$-tree is a $k$-clique; thus, $Z$ has to be the 3 -cycle $\left(w_{p}, w_{q}, v_{k}\right)$. The edge $\left(w_{p}, w_{q}\right)$ cannot be an $n$-edge, since otherwise we would have a cycle in $T_{n}$. So either $\left(w_{q}, w_{p}\right) \in T_{1}$ or $\left(w_{p}, w_{q}\right) \in T_{2}$.

Proof for (b): Mondal et al. 21] have shown that this is true for every triangle in a planar 3-tree.

Proof for (c): The vertices have to lie inside the triangle $\left(w_{p}, w_{q}, v_{k}\right)$ since otherwise their outgoing $n$-edge to $v_{k}$ would cross the edge $\left(w_{p}, w_{q}\right)$. Further, since the subgraph inside $\left(w_{p}, w_{q}, v_{k}\right)$ is again a planar 3 -tree, $w_{p}, w_{q}$, and $v_{k}$ are the roots of the corresponding Schnyder realizer; $\left(w_{p+1}, w_{p}\right) \in T_{1}$ and $\left(w_{q-1}, w_{q}\right) \in T_{2}$ follows immediately.

Given some drawing $\Gamma$ and two points $a$ and $b$ (that are not necessarily a part of $\Gamma$ ), we say that $a$ sees $b$ if and only if the straight-line segment between $a$ and $b$ is interiordisjoint from $\Gamma$. If two points $p$ and $q$ have the same $x$-coordinate and $p$ lies above $q$, then we define slope $(p, q)=-\infty$ and $\operatorname{slope}(q, p)=+\infty$. The following lemma was proven by Durocher and Mondal [11]; see Fig. 7 for an illustration.

Lemma 3 ([11]). Let $a_{1}, \ldots, a_{m}$ be a strictly $x$-monotone polygonal chain $C$. Let $p$ be a point above $C$ such that the segments $a_{1} p$ and $a_{m} p$ do not intersect $C$ except at $a_{1}$ and $a_{m}$. If the positive slopes of the edges of $C$ are smaller than slope $\left(a_{1}, p\right)$, and the negative slopes of the edges of $C$ are greater than slope $\left(p, a_{m}\right)$, then every $a_{i}$ sees $p$.

Note that the first condition trivially holds if all slopes of $C$ are non-positive and that the second condition trivially holds if all slopes of $C$ are non-negative.

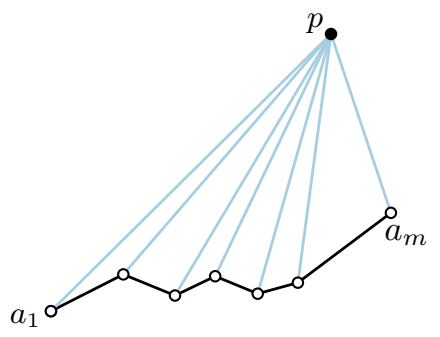

Figure 7: Illustrations of Lemma 3. 


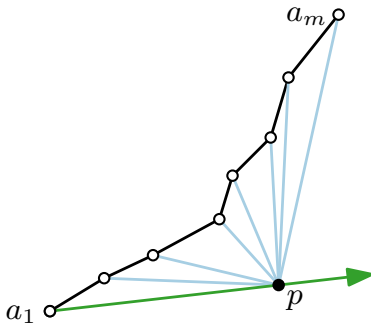

(a) $\Gamma$

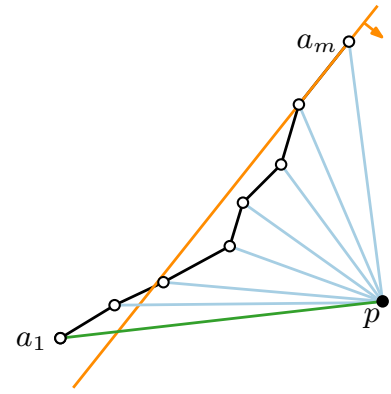

(b) $\Gamma^{\prime}$

Figure 8: Illustrations of Lemma 4 .

We will further need the following lemma which relies on Lemma 3.

Lemma 4. Let $a_{1}, \ldots, a_{m}$ be a strictly $x$-monotone polygonal chain $C$. Let $p$ be a point below $C$ that is connected to $a_{1}, \ldots, a_{m}$ such that $\operatorname{slope}\left(a_{1}, p\right) \geq 0$ and $\operatorname{slope}\left(a_{i}, a_{i+1}\right)>$ slope $\left(a_{1}, p\right)$ for $i=1, \ldots, m-1$. Then the resulting drawing $\Gamma$ is planar. Furthermore, assume that $p$ sees $a_{1}$ and $a_{m}$. If we move $p$ to a point on the ray emanating from $p$ with slope slope $\left(a_{1}, p\right)$, then the resulting drawing $\Gamma^{\prime}$ preserves the embedding of $\Gamma$.

Proof. We first prove that $\Gamma$ is planar. To this end, we first mirror $\Gamma$ at the $x$-axis to obtain a drawing $\Gamma^{*}$. Then $a_{1}, \ldots, a_{m}$ is a strictly $x$-monotone polygonal chain $C$ without positive slopes and $p$ is a point above $C$ such that all (negative) slope of $C$ are smaller than slope $\left(a_{1}, p\right)$. Then by Lemma $3 \Gamma^{*}$ and, hence, $\Gamma$ is planar.

Now we prove that $\Gamma^{\prime}$ preserves the embedding of $\Gamma$. To change the embedding, at least one triangle $\left(a_{i}, a_{i+1}, p\right)$ has to change its orientation, that is, $p$ moves across the supporting line of some $\left(a_{i}, a_{i+1}\right)$. Recall that for all $1 \leq i<m$ we have slope $\left(a_{i}, a_{i+1}\right)>$ slope $\left(a_{1}, p\right)=\operatorname{slope}\left(a_{1}, p^{\prime}\right)$ (see Fig. 8). Since we increase the distance from $a_{1}$ when moving from $p$ to $p^{\prime}$ (and due to the slopes), we also increase the distance to $\left(a_{i}, a_{i+1}\right)$. Thus, both $p$ and $p^{\prime}$ lie in the same halfplane defined by the supporting line of $\left(a_{i}, a_{i+1}\right)$. This proves the lemma.

Overview and notation. The main idea of the algorithm is as follows. We inductively place the vertices according to the canonical order $\sigma=\left(v_{1}, \ldots, v_{n}\right)$ and refer to the step in which vertex $v_{k}$ is placed as step $k$. By the choice of the canonical ordering, when we place vertex $v_{k}$, its parent $v_{\ell}$ in $T_{1}$ has already been placed. If $v_{k}$ is the first child of $v_{\ell}$ in $T_{1}$, then we place $v_{k}$ such that the edge $\left(v_{\ell}, v_{k}\right)$ has the same slope as the edge from $v_{\ell}$ to its parent in $T_{1}$, so that both edges are drawn with one segment. This way, we obtain a drawing of $G$ in which the edges of $T_{1}$ are drawn with exactly one segment for every leaf of $T_{1}$.

In order to be able to do this placement, we have to maintain a series of invariants which rely on additional notation; see Fig. 9. For each vertex $v_{i}$, the 1-out-slope $\operatorname{out}_{1}(i)$ is the slope of its outgoing 1-edge, the 2-out-slope out $_{2}(i)$ is the slope of its outgoing 2-edge, and the in-slope in $(i)$ is the highest slope of the incoming 1-edges in the current drawing. Further, we denote by $\operatorname{par}_{1}(i)$ the 1-parent of $v_{i}$ and by $\operatorname{par}_{2}(i)$ the 2-parent of $v_{i}$. For two vertices $v_{i}, v_{j}$, we denote by $\operatorname{lca}(i, j)$ the lowest common ancestor of $v_{i}$ and $v_{j}$ in $T_{1}$. For an edge $\left(v_{i}, v_{j}\right)$ we call the closed region bounded by $\left(v_{i}, v_{j}\right)$, the path $\left(v_{i}, \ldots\right.$, lca $\left.(i, j)\right)$, and the path $\left(v_{j}, \ldots\right.$, lca $\left.(i, j)\right)$ the domain $\operatorname{dom}(i, j)$ of $\left(v_{i}, v_{j}\right)$. For each step $k$, we denote by $\lambda_{k}$ the number of leafs in the currently drawn subtree of $T_{1}$, by $s_{k}$ 


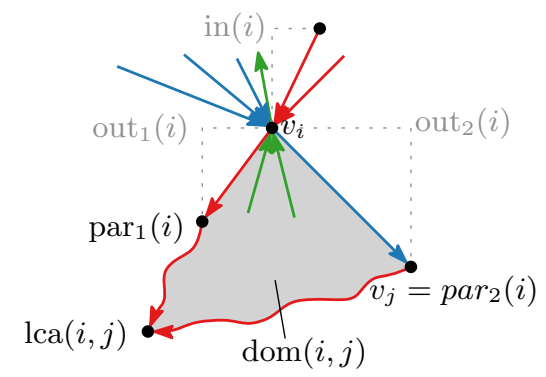

Figure 9: Definitions for the drawing algorithm for planar 3-trees.

the number of segments that are used to draw the current subtree of $T_{1}$, and by $\eta_{k}$ the highest slope of the 1-edges in the current drawing. We denote by $C_{k}^{\rightarrow}$ the part of the contour $C_{k}$ between $v_{k}$ and $v_{2}$.

Before describing the invariants and the algorithm in detail, we first show the following property of $C_{k} \rightarrow$

Lemma 5. The edges on $C_{k} \rightarrow$ are exactly the path from $v_{k}$ to $v_{2}$ in $T_{2}$, and $\operatorname{par}_{2}(k) \in C_{k-1}$.

Proof. We proof the lemma by induction. For $k=3$, we have that $C_{k}=\left(v_{3}, v_{2}\right)$ with $\operatorname{par}_{2}(3)=v_{2}$, so the lemma holds. For $k>3$, recall that we chose the canonical order induced by $T_{2}$ in clockwise pre-order. Hence, either $v_{k-1}$ is the parent of $v_{k}$ in $T_{2}$, or they have a common ancestor $v_{\ell}$ with $\ell<k-1$ in $T_{2}$. In the former case, $\operatorname{par}_{2}(k)=v_{k-1} \in C_{k-1}$ and $C_{k} \rightarrow=v_{k} \circ C_{k-1}^{\rightarrow}$, so the lemma holds by induction. In the latter case, we in fact have $\operatorname{par}_{2}(k)=v_{\ell}$. By induction, $C_{k-1}^{\rightarrow}=\left(v_{k-1}, \ldots, v_{\ell}, \ldots, v_{2}\right)$, so $\operatorname{par}_{2}(k) \in C_{k-1}^{\rightarrow}$ and $C_{k}^{\rightarrow}=\left(v_{k}, v_{\ell}, \ldots, v_{2}\right)$.

Invariants. After each step $k \geq 3$, we maintain the following invariants.

(I1) The contour $C_{k}$ is a strictly $x$-monotone polygonal chain; the $x$-coordinates along $C_{k} \rightarrow$ increase by exactly 1 per vertex.

(I2) The 1-edges are drawn with $s_{k}=\lambda_{k}$ segments in total with integer slopes between 1 and $\eta_{k} \leq \lambda_{k}$

(I3) For each $\left(v_{i}, v_{j}\right) \in C_{k} \rightarrow$ and for each 1-edge $e \neq\left(\operatorname{par}_{1}(j), v_{j}\right)$ in $\operatorname{dom}(i, j)$ it holds that $\operatorname{slope}(e)>\operatorname{out}_{1}(j)$.

(I4) For each $v_{i} \in C_{k}$, out $_{1}(i)>\operatorname{out}_{2}(i)$.

(I5) The current drawing is crossing-free and for each $\left(v_{i}, v_{j}\right) \in T_{n}$, we have slope $\left(v_{i}, v_{j}\right)>$ $\operatorname{out}_{1}(j)$.

(I6) Vertex $v_{1}$ is placed at coordinate $(0,0), v_{2}$ is placed at coordinate $(k-1,0)$, and every vertex lies inside the rectangle $(0,0) \times\left(k-1,(k-1) \lambda_{k}\right)$.

The algorithm. The algorithm starts with placing $v_{1}$ at $(0,0), v_{2}$ at $(2,0)$, and $v_{3}$ at $(1,1)$. Obviously, all invariants (I1) (I6) hold. In step $k>3$, the algorithm proceeds in two steps. Recall that $v_{k}$ is a neighbor of all vertices on the contour between $v_{l}=\operatorname{par}_{1}(k)$ and $v_{r}=\operatorname{par}_{2}(k)$.

Step 1: Insertion. In the insertion step, $v_{k}$ is placed with the same $x$-coordinate as $v_{r}$. We distinguish between three cases to obtain the $y$-coordinate of $v_{k}$; see Fig. 10 for an illustration.

(i) If no incoming 1-edge of $v_{l}$ has been drawn yet, then we draw the edge $\left(v_{l}, v_{k}\right)$ with slope $\operatorname{out}_{1}(l)$. 


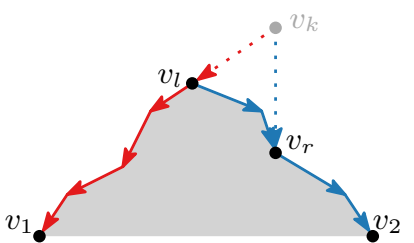

(a) Case (i)

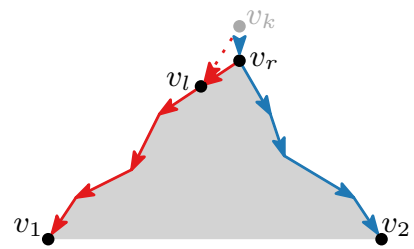

(b) Case (ii)

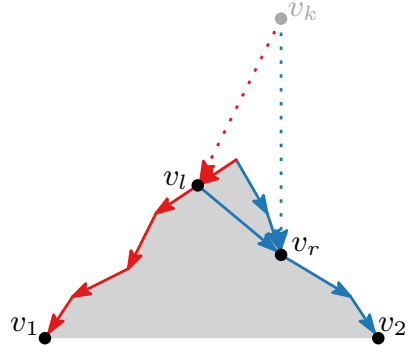

(c) Case (iii)

Figure 10: Inserting vertex $v_{k}$ while maintaining invariant (I5)

(ii) If $v_{l}$ already has an incoming 1-edge and $v_{l}$ and $v_{r}$ are the only neighbors of $v_{k}$ in the current drawing, then we draw the edge $\left(v_{l}, v_{k}\right)$ with slope in $(l)+1$.

(iii) Otherwise, we draw the edge $\left(v_{l}, v_{k}\right)$ with slope $\eta_{k-1}+1$.

Note that this does not maintain invariant (I1).

Step 2: Shifting. In the shifting step, the vertices between $v_{r}$ and $v_{2}$ on the contour $C_{k}$ have to be shifted to the right without increasing the number of segments $s_{k}$ used to draw $T_{1}$. To this end, we iteratively extend the outgoing 1-edge of these vertices, starting with $v_{r}$, to increase their $x$-coordinates all by 1 ; see Fig. 11 . This procedure places the vertices on the grid since the slopes of the extended edges are all integer by invariant (I2).

In the following, we will show that every planar 3-tree admits a straight-line drawing that uses at most $(8 n-17) / 3$ segments on an $O(n) \times O\left(n^{2}\right)$ grid, and that this drawing can be computed in $O\left(n^{2}\right)$ time. We have to show that, after each step $k>3$, all invariants (I1) (I6) hold. We denote by $x(v)$ the $x$-coordinate of the vertex $v$, and similarly, by $y(v)$ the $y$-coordinate of $v$.

Invariant (I1). The contour in step $k$ is $C_{k}=\left(v_{1}, \ldots, v_{l}, v_{k}, v_{r}, \ldots, v_{2}\right)$. By induction, $\left(v_{1}, \ldots, v_{l}, v_{r}, \ldots, v_{2}\right)$ is strictly $x$-monotone. We place $v_{k}$ above $v_{r}$ and move all vertices from $v_{r}$ to $v_{2}$ to the right by one unit, so $x\left(v_{l}\right)<x\left(v_{k}\right)=x\left(v_{r}\right)-1$ and $C_{k}$ is strictly $x$-monotone. By Lemma 5, $v_{r}$ lies on $C_{k-1}^{\rightarrow}$, so the $x$-coordinates along $\left(v_{r}, \ldots, v_{2}\right)$ increase by 1 per vertex; since $x\left(v_{r}\right)=x\left(v_{k}\right)$, the same holds for $C_{k}^{\rightarrow}$.

Invariant (I2). In case (i) of the insertion step, $v_{l}$ changes from a leaf to a non-leaf, while $v_{k}$ is added as a leaf. Since $\left(v_{l}, v_{k}\right)$ is drawn with the same slope as the outgoing 1-edge of $v_{l}$, we have $s_{k}=s_{k-1}=\lambda_{k-1}=\lambda_{k} \geq \eta_{k}$. In cases (ii)] and (iii) of the insertion step, $v_{l}$ was not a leaf before and a new integer slope is used to draw $\left(v_{l}, v_{k}\right)$, so we have
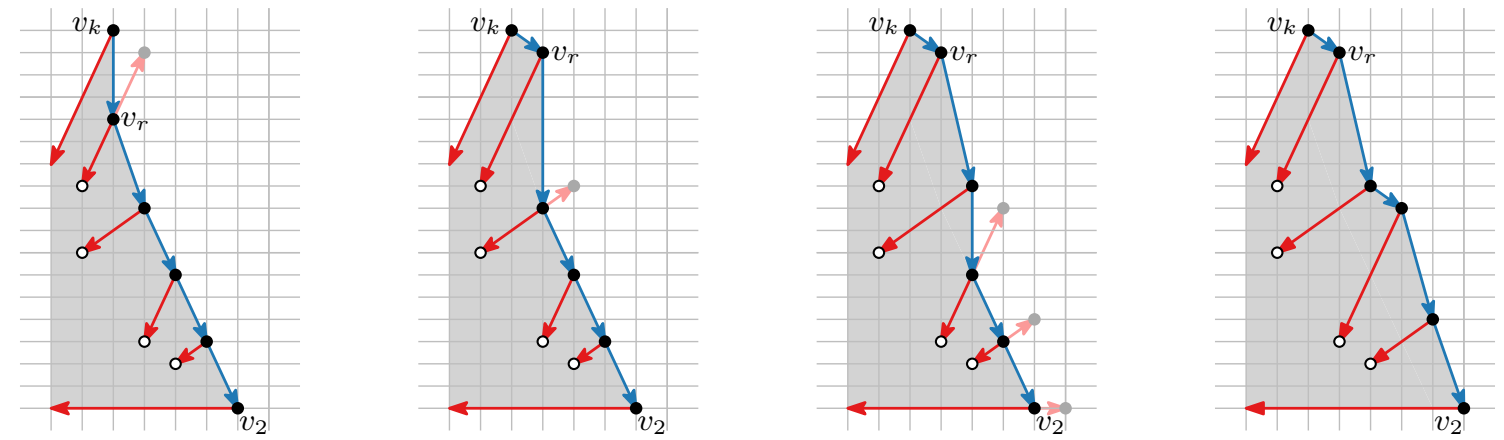

Figure 11: Shifting $v_{r}, \ldots, v_{2}$ along their outgoing 1-edge. 


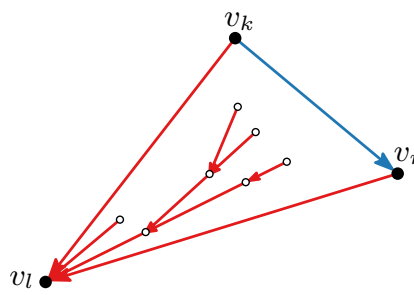

(a) Case $\left(v_{r}, v_{l}\right) \in T_{1}$

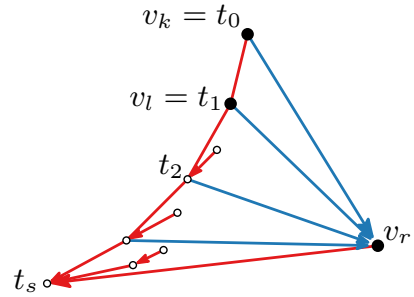

(b) Case $\left(v_{l}, v_{r}\right) \in T_{2}$

Figure 12: The domain $\operatorname{dom}(k, r)$ as in invariant (I3).

$s_{k}=s_{k-1}+1=\lambda_{k-1}+1=\lambda_{k}$; since the maximum slope increases by at most one, we have $\eta_{k} \leq \eta_{k-1}+1 \leq \lambda_{k}$. For the shift step, as the vertices on $C_{k}^{\rightarrow}$ have no incoming 1-edges, and the slopes of their outgoing 1-edges do not change, the number of segments remains the same.

Invariant (I3), We have to only address the insertion step, since the shifting step does not affect the relevant slopes. Since the slopes of the 1-edges do not change, and since the edges on $\left(v_{r}, \ldots, v_{2}\right)$ lie on $C_{k-1}^{\rightarrow}$ by Lemma 5 , the invariant holds for edges on $\left(v_{r}, \ldots, v_{2}\right)$ by induction; hence, it suffices to show the invariant for $\left(v_{k}, v_{r}\right)$. By Lemma 2(a), the edge $\left(v_{l}, v_{r}\right)$ exists in $T_{1}$ or $T_{2}$. We distinguish between two cases.

Case 1: $\left(v_{r}, v_{l}\right) \in T_{1}$; see Fig. 12a. It immediately follows that lca $(k, r)=v_{l}=\operatorname{par}_{1}(k)$. Hence, the domain $\operatorname{dom}(k, r)$ is bounded by the triangle $\left(v_{l}, v_{r}, v_{k}\right)$, which is a planar 3 -tree by Lemma $2(\mathrm{~b})$ with $v_{l}$ as the root of $T_{1}$. By construction, the edge $\left(v_{r}, v_{l}\right)$ has the smallest slope of all incoming 1-edges of $v_{l}$ within the domain. In particular, by construction, the first incoming 1-edge of each vertex is assigned the same slope as the outgoing 1-edge, while all other incoming 1-edges are assigned higher slopes. Thus, all 1-edges in the domain must have higher slopes than $\left(v_{r}, v_{l}\right)$.

Case 2: $\left(v_{l}, v_{r}\right) \in T_{2}$; see Fig. 12b. Consider the unique path from $v_{k}$ to $v_{1}$ in $T_{1}$. Denote the vertices on this path by $t_{0}, t_{1}, t_{2}, \ldots, t_{q}$ with $t_{0}=v_{k}, t_{1}=v_{l}$, and $t_{q}=v_{1}$. By Lemma 2, $t_{1}=v_{l}$ is connected to $v_{r}$ and $\left(t_{1}, v_{r}\right) \in T_{1}$ or $\left(t_{1}, v_{r}\right) \in T_{2}$. If $\left(t_{1}, v_{r}\right) \in T_{2}$, then consider the drawing of $G_{l}$ after step $l$ of the algorithm. In this step, $t_{1}=v_{l}$ was placed, $v_{r}$ is its 2-parent, and $t_{2}$ is its 1-parent. Hence, by Lemma $2, t_{2}$ is connected to $v_{r}$ and $\left(t_{2}, v_{r}\right) \in T_{1}$ or $\left(t_{2}, v_{r}\right) \in T_{2}$. It follows by induction that, as long as $\left(t_{i}, v_{r}\right) \in T_{2}$, then $\left(t_{i+1}, v_{r}\right)$ exists.

Let $t_{s}$ be the first vertex on the path $t_{0}, t_{1}, \ldots, t_{q}$ such that $\left(t_{s}, v_{r}\right)$ exists but is not a 2-edge. Since $t_{q}=v_{1}$ but $v_{1}$ has no 2-parent, this vertex exists; since $\left(t_{0}=v_{k}, v_{r}\right),\left(t_{1}=\right.$ $\left.v_{l}, v_{r}\right) \in T_{2}$, we have $s>1$. By choice of $t_{s}$, we have that $\left(t_{s-1}, t_{s}\right) \in T_{1}$ and $\left(t_{s-1}, v_{r}\right) \in T_{2}$; hence, by Lemma 2(a), the edge $\left(v_{r}, t_{s}\right)$ exists and since $\left(t_{s}, v_{r}\right) \notin T_{2}$ it has to be a 1 edge. Hence, we have $\operatorname{par}_{1}(r)=t_{s}=\operatorname{lca}(k, r)$. The domain $\operatorname{dom}(k, r)$ is bounded by the edges $\left(v_{k}, v_{r}\right),\left(v_{r}, t_{s}\right)$, and the path $\left(t_{0}:=v_{k}, t_{1}, \ldots, t_{s}\right)$. By Lemma $2(\mathrm{~b})$, the domain can be divided into $s$ planar 3 -trees $\left(t_{i}, v_{r}, t_{i-1}\right), 1 \leq i \leq s$. For the triangle $\left(t_{s}, v_{r}, t_{s-1}\right)$, the argument from Case 1 can be directly applied; for $i<s$, we can use the same argument to show that no 1-edge has a higher slope than $\left(t_{i}, t_{i+1}\right)$, which proves the invariant.

Invariant (I4), Let $v_{i} \in C_{k}^{\rightarrow}$. First, consider the case that $v_{i}=v_{k}$; see Fig. 13a. In the insertion step, $v_{k}$ is placed at the same $x$-coordinate as $v_{r}$. In the shifting step, $v_{r}$ is moved to the right by one column and upwards by $\operatorname{out}_{1}(r)$ rows; hence, we have $y\left(v_{r}\right)<$ $y\left(v_{k}\right)+\operatorname{out}_{1}(r)$. Since $\operatorname{dom}(k, r)$ contains $v_{l}=\operatorname{par}_{1}(k)$, it follows from invariant (I3) that $\operatorname{out}_{2}(k)=y\left(v_{r}\right)-y\left(v_{k}\right)<\operatorname{out}_{1}(r)<\operatorname{out}_{1}(k)$. 


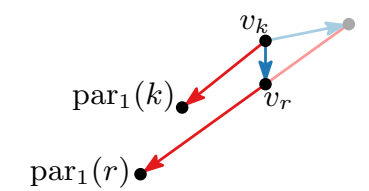

(a) $i=k$ and $v_{r}=\operatorname{par}_{2}(k)$

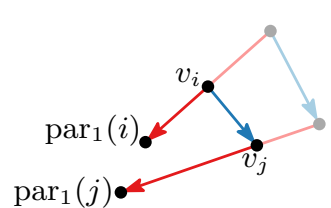

(b) $i \neq k$ and $v_{j}=\operatorname{par}_{2}(i)$

Figure 13: Shifting $\operatorname{par}_{2}(i)$ while maintaining invariant (I4).

Now, consider the case that $v_{2} \neq v_{i} \neq v_{k}$; see Fig. $13 \mathrm{~b}$. Let $v_{j}=\operatorname{par}_{2}(i)$. By Lemma 5 , $\left(v_{i}, v_{j}\right) \in C_{k-1}^{\rightarrow}$, so we had $\operatorname{out}_{1}(i)>$ out $_{2}(i)$ before the shifting step of the algorithm. In the shifting step, out ${ }_{1}(i)$ does not change. However, $v_{i}$ and $v_{j}$ are shifted to the right by one column and upwards by out $(i)$ rows and $\operatorname{out}_{1}(j)$ rows, respectively; hence, $\operatorname{out}_{2}(i)$ increases by $\operatorname{out}_{1}(j)-\operatorname{out}_{1}(i)$. Since $\operatorname{dom}(i, j)$ contains $v_{l}=\operatorname{par}_{1}(i)$, it follows from invariant (I3) that $\operatorname{out}_{1}(j)<$ out $_{1}(i)$. This implies that $\operatorname{out}_{2}(i)$ becomes smaller by the shifting step, so out $1(i)>$ out $_{2}(i)$ is maintained.

Invariant (I5). First, consider the drawing after the insertion step, but before the shifting step. By induction, the drawing was crossing-free before this step. Since no existing edge is modified, each crossing has to involve an edge of $v_{k}$. We will use Lemma 3 to show that $v_{k}$ sees all its neighbors. By invariant (I1), the neighbors of $v_{k}$ lie on a strictly $x$-monotone polygonal chain. Because of slope $\left(v_{k}, v_{r}\right)=-\infty$, it suffices to show that the edges between $v_{l}$ and $v_{r}$ on the contour have smaller slope than slope $\left(v_{l}, v_{k}\right)$.

Consider case (i) of the insertion step: $v_{l}$ has no incoming 1-edge; see Fig. 10a. Then, $\left(v_{l}, v_{r}\right) \in C_{k-1}$; otherwise, we get a contradiction from Lemma 2(c). Hence, $v_{l}$ and $v_{r}$ are the only neighbors of $v_{k}$ and we have to show only that slope $\left(v_{k}, v_{l}\right)>\operatorname{slope}\left(v_{l}, v_{r}\right)$. By construction and invariant (I4), we have slope $\left(v_{k}, v_{l}\right)=\operatorname{out}_{1}(l)>\operatorname{out}_{2}(l)=\operatorname{slope}\left(v_{l}, v_{r}\right)$.

Consider case (ii) of the insertion step: $v_{l}$ already has an incoming 1-edge and $v_{l}$ and $v_{r}$ are the only neighbors of $v_{k}$; see Fig. $10 \mathrm{~b}$. In particular, that means that $\left(v_{r}, v_{l}\right) \in T_{1}$ and, by construction, $\operatorname{slope}\left(v_{k}, v_{l}\right)=\operatorname{in}(l)+1>\operatorname{in}(l) \geq \operatorname{slope}\left(v_{r}, v_{l}\right)$.

Consider case (iii) of the insertion step: $v_{l}$ already has an incoming 1-edge and $\left(v_{l}, v_{r}\right) \notin$ $C_{k-1}$; see Fig. 10c. In this case, $\left(v_{k}, v_{l}\right)$ is drawn with slope $\left(v_{k}, v_{l}\right)=\eta_{k-1}+1$, so every 1-edge on the contour between $v_{l}$ and $v_{r}$ has lower slope than $\left(v_{k}, v_{l}\right)$. By invariant (I2) and (I4), the 2-edges on the contour between $v_{l}$ and $v_{r}$ also cannot have higher slope than $\eta_{k-1}$.

Hence, we can use Lemma 3 in each case of the insertion step to show that the drawing remains planar. The second part of the invariant, for each $\left(v_{i}, v_{j}\right) \in T_{n}$, slope $\left(v_{i}, v_{j}\right)>$ $\operatorname{out}_{1}(j)$, holds for each vertex but $v_{k}$ by induction. Since the edges of $v_{k}$ are planar and $v_{k}$ is placed vertically above $v_{r}=\operatorname{par}_{2}(k)$, all edges $\left(v_{i}, v_{k}\right) \in T_{n}$ have positive slope with $x\left(v_{i}\right)>x\left(v_{l}\right)$, so $\operatorname{slope}\left(v_{i}, v_{k}\right)>\operatorname{out}_{1}(k)$.

Now, consider the drawing after the shifting step. We shift the vertices between $v_{r}$ and $v_{2}$ one by one on the contour $C_{k} \rightarrow$ and show that, after each step, the invariant holds. Let $v_{t}$ be the vertex next to shift, let $v_{s}$ be its predecessor and let $v_{u}=\operatorname{par}_{2}(t)$ be its successor on the contour. Since either $v_{s}=v_{k}$ or $v_{s}$ has been shifted in the previous step, the edge $\left(v_{s}, v_{t}\right)$ is drawn vertically and the edge $\left(v_{t}, v_{u}\right)$ has slope out $2(t)$. Since $\left(v_{t}, \operatorname{par}_{1}(t)\right)$ lies on the boundary of both domains $\operatorname{dom}(s, t)$ and $\operatorname{dom}(t, u)$, the union of these domains contains all edges incident to $v_{t}$ and all $n$-edges of $v_{t}$ lie in $\operatorname{dom}(t, u)$. We will now show that both domains remain planar and for all $\left(v_{i}, v_{t}\right) \in T_{n}$, slope $\left(v_{i}, v_{t}\right)>\operatorname{out}_{1}(t)$.

Consider the domain $\operatorname{dom}(s, t)$; see Fig. 14a. By the Schnyder realizer properties, all edges incident to $v_{t}\left(\right.$ except $\left.\left(v_{t}, \operatorname{par}_{1}(t)\right)\right)$ are 2-edges. Let $\operatorname{par}_{1}(t)=a_{1}, \ldots, a_{m}=v_{s}$ 


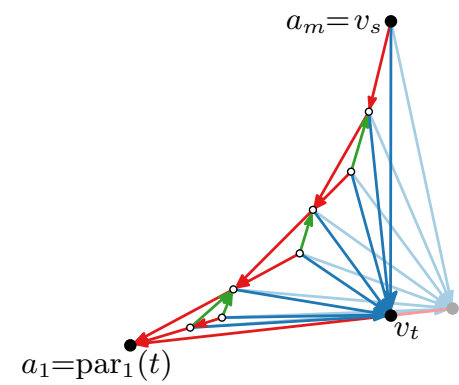

(a) Maintaining the invariant in $\operatorname{dom}(s, t)$

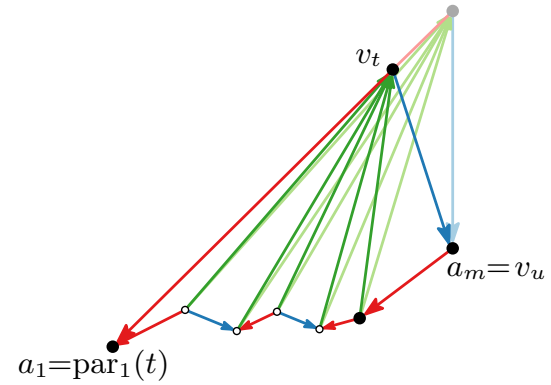

(b) Maintaining the invariant in $\operatorname{dom}(t, u)$

Figure 14: Shifting vertex $v_{t}$ while maintaining invariant (I5)

be the path through the neighbors of $v_{t}$. All edges on this path are 1-edges or $n$-edges (otherwise $T_{2}$ would not be a tree). By invariant (I3), all 1-edges on this path have slope higher than out $1(t)$. By induction and by invariant (I3), for each $n$-edge $\left(a_{i-1}, a_{i}\right)$ on this path, we have slope $\left(a_{i-1}, a_{i}\right)>\operatorname{out}_{1}(i)>\operatorname{out}_{1}(t)$. As out $(t)>0$, the path $a_{1}, \ldots, a_{m}$ is an $x$-monotone chain without negative slopes. Hence, we can apply Lemma 4 to prove that the new position of $v_{t}$ preserves the embedding, so the drawing remains planar.

Now, consider the domain $\operatorname{dom}(t, u)$; see Fig. 14b. By the Schnyder realizer properties, all edges incident to $v_{t}$ (except $\left.\left(v_{t}, \operatorname{par}_{2}(t)\right)\right)$ are $n$-edges. Let $\operatorname{par}_{1}(t)=a_{1}, \ldots, a_{m}=v_{u}$ be the path through the neighbors of $v_{t}$. All edges on this path are 1-edges or 2-edges (otherwise $T_{n}$ would not be a tree). If $m=2$, then there is no $n$-edge in $\operatorname{dom}(t, u)$. By invariant (I3), we have out $1(t)>\operatorname{slope}\left(a_{1}, a_{2}\right)=\operatorname{out}_{1}(u)$ and we can move $v_{t}$ in direction $\operatorname{par}_{1}(t) v_{t}$ without changing the embedding. If $m>2$, we look back on step $t$ of the algorithm. Observe that $a_{1}, \ldots, a_{m}$ were the neighbors of $v_{t}$ on the contour $C_{t-1}$. Since case (i) and (ii) imply $m=2, v_{t}$ had to be inserted with case (iii). By construction, the edge $\left(\operatorname{par}_{1}(t), v_{t}\right)$ was inserted with a higher slope than all 1-edges and, by invariant (I3), also all 2-edges on the contour. Further, as $a_{1}, \ldots, a_{m-1}$ were removed from the contour and the algorithm only changes the drawing of edges incident to a vertex on the contour, the edges $\left(a_{i}, a_{i+1}\right)$ for $1 \leq i \leq m-2$ were not changed afterward. Thus, we still have $\operatorname{out}_{1}(t)>\operatorname{slope}\left(a_{i}, a_{i+1}\right), 1 \leq i \leq m-2$. Additionally, by invariant (I3), also $\operatorname{out}_{1}(t)>$ slope $\left(a_{m-1}, a_{m}\right)$ holds, so by Lemma 3 we can place $v_{t}$ above $v_{u}$ with slope out ${ }_{1}(t)$ without crossings (since we obtain slope $\left.\left(v_{t}, v_{u}\right)=-\infty\right)$. The movement of $v_{t}$ to the right and the planarity maintain that $\operatorname{slope}\left(a_{i}, v_{t}\right)>\operatorname{out}_{1}(t), 2 \leq i \leq m-1$. This establishes invariant (I5)

Invariant (I6). Since $v_{1}$ is never moved, it remains at $(0,0)$. Vertex $v_{2}$ is moved $k-3$ times to the right by one column, so it is located at $((k-3)+2,0)=(k-1,0)$. As $v_{k}$ is not placed to the right of $v_{2}$ and no vertex is moved to the right by more than one column, the maximum $x$-coordinate of all vertices is $k-1$. The contour consists of only 1-edges and 2-edges; by invariants (I4) and (I2), their maximum slope is $\lambda_{k}$. Hence, the maximum $y$-coordinate of all vertices is at most $(k-1) \lambda_{k}$.

This proves the correctness of all invariants. We will now analyze the number of segments used by the algorithm. By Invariant (I2), the 1-edges are drawn with $s_{n}=\lambda_{n}$ slopes, where $\lambda_{n}$ is equals to the number of leaves in $T_{1}$. Recall that the number of leaves in the Schnyder realizer is at most $2 n-5$ [1] and that we chose $T_{1}$ as the canonical ordering tree with the fewest leaves, so we have $\lambda_{n} \leq(2 n-5) / 3$. The trees $T_{2}$ and $T_{n}$ contain $n-1$ vertices, so they are both drawn with at most $n-2$ segments. Hence, the total number of segments is at most $(2 n-5) / 3+(n-2)+(n-2)=(8 n-17) / 3$. 


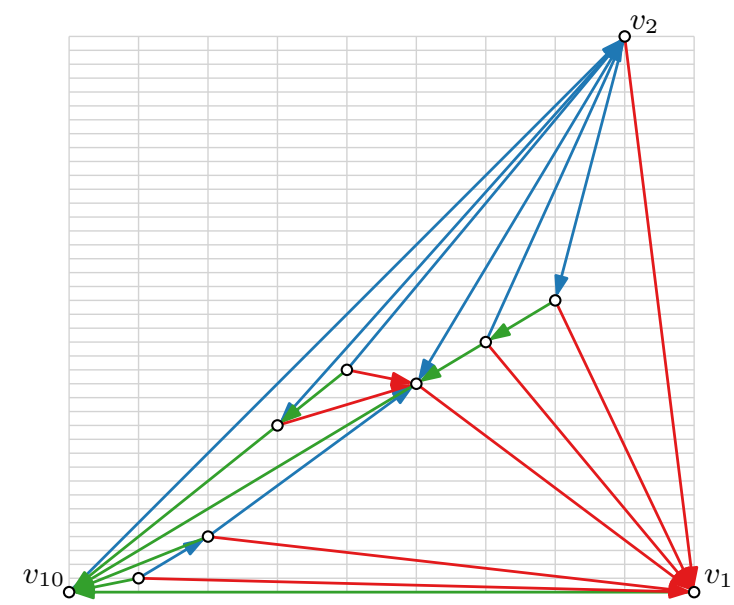

Figure 15: The drawing produced by our algorithm for the planar 3-tree depicted in Figure 5

It remains to show the running time of the algorithm. Both the Schnyder realizer and the canonical order can be computed in linear time [23, 9]. The insertion step can be handled in constant time by storing at each vertex the highest slope of its incoming 1-edges (if it exists) and updating the value $\eta_{k}$ at each step. In the shifting step, we have to move all vertices on the contour between the placed vertex and $v_{2}$ by stretching their outgoing 1-edges. Since there are at most $n-2$ vertices on this part of the contour and we have $n-2$ steps, this takes $O\left(n^{2}\right)$ time in total. It is not clear whether we can use an approach similar to the one by Chrobak and Payne [6] in order to reduce the running time of the shifting step as the vertices have to be moved in two directions instead of only one and since we have to know the exact coordinates and slopes at every step.

This proves the following theorem, which is the main result of this section. See Figure 15 for an example.

Theorem 3. Every planar 3-tree admits a straight-line drawing that uses at most ( $8 n-$ 17)/3 segments on an $O(n) \times O\left(n^{2}\right)$ grid. This drawing can be computed in $O\left(n^{2}\right)$ time.

\section{Maximal outerplanar graphs with segments on the grid}

A graph is outerplanar if it can be embedded in the plane with all vertices on one face (called outerface), and it is maximal outerplanar if no edge can be added while preserving outerplanarity. This implies that all interior faces of a maximal outerplanar graph are triangles. Outerplanar graphs have degeneracy 2 [19], that is, every induced subgraph of an outerplanar graph has a vertex with degree at most two. Thus, we find in every maximal outerplanar graph a vertex of degree 2 whose removal (taking away one triangle) results in another maximal outerplanar graph. By this, we gain a deconstruction order (also known as ear-decomposition) that stops with a triangle. Let $G=(V, E)$ be a maximal outerplanar graph and let $\sigma=\left(v_{1}, \ldots, v_{n}\right)$ be the reversed deconstruction order.

Lemma 6. The edges of $G$ can be partitioned into two trees $T_{1}$ and $T_{2}$. Moreover, we can turn $G$ into a planar 3-tree by adding a vertex and edges in the outerface. The additional edges form a tree $T_{n}$. The three trees $T_{1}, T_{2}$ and $T_{n}$ induce a Schnyder realizer.

Proof. We build the graph $G$ according to the reversed deconstruction order. Let $G_{k}$ denote the subgraph of $G$ induced by the set $\left\{v_{1}, v_{2}, \ldots, v_{k}\right\}$. Further, let $G_{k}^{\prime}$ be the graph 

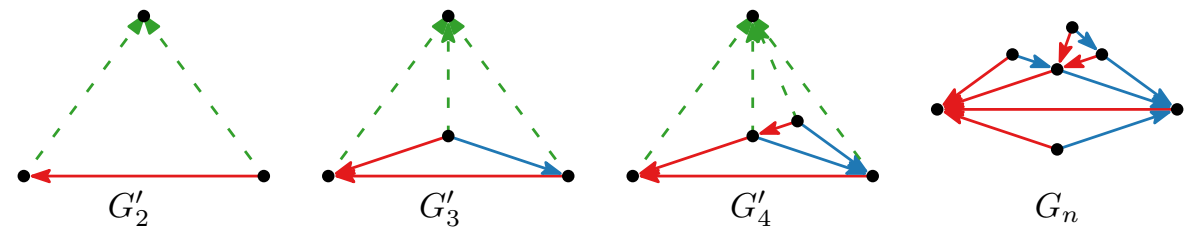

Figure 16: Construction of an maximal outerplanar graph embedded in a planar 3-tree as done in the proof of Lemma 6. The tree $T_{n}$ is dashed.

obtained by adding the vertex $v_{n}$ and the edges $\left(v_{i}, v_{n}\right)$ for all $1 \leq i \leq k$ to $G$. We prove by induction over $k$ that there exists a Schnyder realizer induced by $T_{1}, T_{2}, T_{n}$ for $G_{k}^{\prime}$ such that the trees $T_{1}$ and $T_{2}$ form the graph $G_{k}$ and $G_{k}^{\prime}$ is a planar 3-tree. Note that Felsner and Trotter [13] already proved this lemma without the statement that $G_{k}^{\prime}$ is a planar 3-tree.

For the base case $k=2$, our hypothesis is certainly true; see $G_{2}^{\prime}$ in Fig. 16. Assume our assumption holds for some $k$. In order to obtain $G_{k+1}$ from $G_{k}$, we have to add the vertex $v_{k+1}$ and two incident edges $\left(v_{i}, v_{k+1}\right)$ and $\left(v_{j}, v_{k+1}\right)$. Assume that $v_{i}$ is left of $v_{j}$ on $C_{k}$. We add $\left(v_{i}, v_{k+1}\right)$ to $T_{1}$ and $\left(v_{j}, v_{k+1}\right)$ to $T_{2}$. This is safe since we cannot create a cycle in any of the trees. There is another a new edge $\left(v_{k+1}, v_{n}\right)$ in $G_{k+1}^{\prime}$ which we add to $T_{n}$. Again, no cycle can be created. The three outgoing tree edges at $v_{i}$ form three wedges (unless $v_{i}$ is the root of $T_{1}$ ). The new ingoing 1-edge $\left(v_{i}, v_{k+1}\right)$ lies in the wedge bounded by the outgoing 2-edge and the outgoing $n$-edge. For the edge $\left(v_{j}, v_{k+1}\right)$, we can argue analogously. Hence, the three trees induce a Schnyder realizer; see also Fig. 16. Moreover, the graphs $G_{k}^{\prime}$ and $G_{k+1}^{\prime}$ differ exactly by the vertex $v_{k+1}$ that has been stacked into a triangular face of $G_{k}^{\prime}$; thus, since $G_{k}^{\prime}$ is a planar 3-tree, so is $G_{k+1}^{\prime}$. We now have proven the induction hypothesis for $k+1$. To obtain the statement of the lemma, we take the Schnyder realizer for the graph $G_{n-1}^{\prime}$ and move the edge $\left(v_{1}, v_{n}\right)$ from $T_{n}$ to $T_{1}$ and the edge $\left(v_{2}, v_{n}\right)$ from $T_{n}$ to $T_{2}$. Now $T_{1}$ and $T_{2}$ form $G$, and all three trees induce the Schnyder realizer of a planar 3-tree.

We can now rely on our methods developed for the planar 3-trees. The technique used in Theorem 3 produces a drawing of a planar 3-tree in which two of the trees of the Schnyder realizer are drawn with single-edge segments, whereas the third tree uses as many segments as it had leaves.

Consider a drawing according to Theorem 3 of the planar 3-tree introduced in Lemma 6 that contains the maximal outerplanar graph $G$ as an induced subgraph. By deleting $T_{n}$, we obtain a drawing of $G$. Note that in this drawing the outer face is realized as an interior face, which can be avoided (if this is undesired) by repositioning $v_{n}$ accordingly. The Schnyder realizer has $2 n-5$ leaves in total, but $n-3$ of them belong to $T_{n}$. We can assume that $T_{1}$ has the smallest number of leaves, which is at most $n / 2-1$. We need $n-2$ segments for drawing $T_{2}$ (one per edge), and three edges for the triangle $v_{1}, v_{2}, v_{n}$. In total, we have at most $n / 2-1+n-2+3=3 n / 2$ segments. Since the drawing is a subdrawing from our drawing algorithm for planar 3 -trees, we get the same area bound and running time as in the planar 3-tree scenario. We summarize our results in the following theorem.

Theorem 4. Every maximal outerplanar graph admits a straight-line drawing that uses at most $3 n / 2$ segments on an $O(n) \times O\left(n^{2}\right)$ grid. This drawing can be computed in $O\left(n^{2}\right)$ time. 


\section{Triangulations with circular arcs}

In this section, we present an algorithm to draw a triangulation using few circular arcs. An embedded graph is a triangulation (or maximal planar) if every face is a 3-cycle, including the outerface. Our algorithm draws upon ideas for drawing triangulations with line segments by Durocher and Mondal [11] as well as Schulz's algorithm for drawing 3 -connected planar graphs with circular arcs [24].

Similar to Schulz [24, a canonical order $v_{1}, \ldots, v_{n}$ on the vertices of a triangulation is reversed and used to structure our drawing algorithm. We start by drawing $v_{1}, v_{2}$, and $v_{n}$ on a circle; see Fig. 17a. We assume that they are placed as shown and hence refer to the arc connecting $v_{1}$ and $v_{2}$ as the bottom arc. The interior of the circle is the undrawn region $\mathcal{U}$ which we maintain as a strictly convex shape. The vertices incident to $\mathcal{U}$ are referred to as the horizon and denoted $h_{1}, h_{2}, \ldots, h_{k-1}, h_{k}$ in order; see Fig. $17 \mathrm{~b}$. We maintain that $h_{1}=v_{1}$ and $h_{k}=v_{2}$. Initially, we have $k=3$ and $h_{2}=v_{n}$. We iteratively take a vertex $h_{i}$ of the horizon (the latest in the canonical order) to process it. That is, we draw its undrawn neighbors and edges between these, thereby removing $h_{i}$ from the horizon. Note that $h_{i}$ will never be the first or last vertex on the horizon $\left(v_{1}\right.$ or $\left.v_{2}\right)$.

Invariant. We maintain as invariant that each vertex $v$ (except $v_{1}, v_{2}$, and $v_{n}$ ) has a segment $\ell_{v}$ incident from above such that its downward extension intersects the bottom arc strictly between $v_{1}$ and $v_{2}$. Since $\mathcal{U}$ is strictly convex, observe that for any vertex $h$ on the horizon the only intersection points of the line through $\ell_{h}$ with the undrawn region's boundary are this intersection with the bottom arc and $h$ itself.

Processing a vertex. To process a vertex $h_{i}$, we first consider the triangle $h_{i-1} h_{i} h_{i+1}$ : this triangle (except for its corners) is strictly contained in $\mathcal{U}$. We draw a circular arc $A$ from $h_{i-1}$ to $h_{i+1}$ with maximal curvature, but within this triangle; see Fig. 18a. This ensures a plane drawing and maintains a strictly convex undrawn region. Moreover, it ensures that $h_{i}$ can "see" the entire arc $A$.

Vertex $h_{i}$ may have a number of neighbors that were not yet drawn. We dedicate a fraction of the arc $A$ to placing these neighbors, . In particular, this fraction is determined by the intersections of segments $v_{1} h_{i}$ and $v_{2} h_{i}$ with $A$; see Fig. $18 \mathrm{~b}$, By convexity of $\mathcal{U}$, these intersections exist. If $h_{i-1}$ is equal to $v_{1}$, then the intersection for $v_{1} h_{i}$ degenerates to $v_{1}$; similarly, the intersection of $v_{2} h_{i}$ may degenerate to $v_{2}$. We place the neighbors in order along this designated part of $A$, drawing the relevant edges as line segments. This implies that all these neighbors obtain a line segment that extends to intersect the bottom arc, maintaining the invariant. We position one neighbor to be a continuation of segment $\ell_{h_{i}}$, which by the invariant must extend to intersect the designated part of $A$ as well. Two

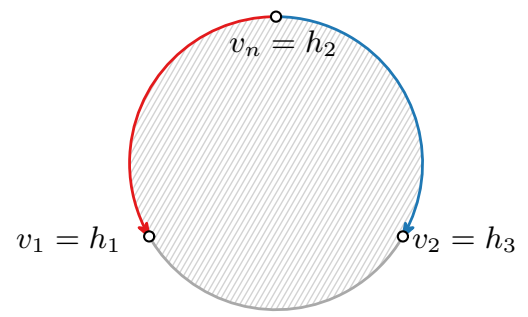

(a) Initial state of the algorithm.

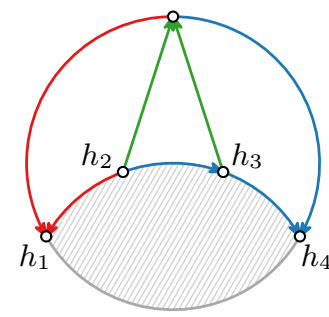

(b) The state after processing $v_{n}$.

Figure 17: Illustration of the algorithm to draw triangulations with few circular arcs. Hatching indicates the undrawn region. 


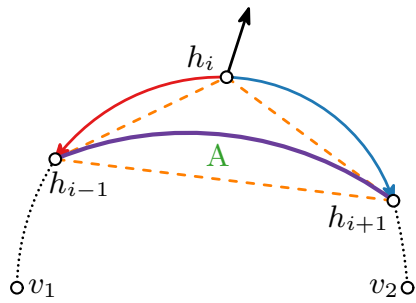

(a)

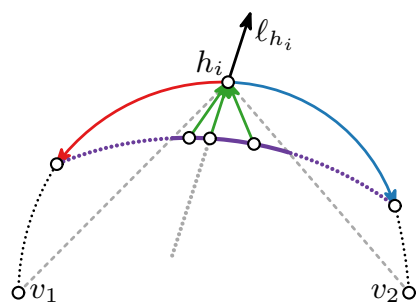

(b)

Figure 18: (a) Arc $A$ lies inside the dashed triangle $h_{i-1} h_{i} h_{i+1}$. (b) Undrawn neighbors of $h_{i}$ are placed on $A$, in the section determined by $v_{1}$ and $v_{2}$. One neighbor is placed to align with $\ell_{h_{i}}$ towards a predecessor of $h_{i}$.

examples of fully drawn graphs are given in Fig. 19. Note that the angular resolution of these drawings decreases very rapidly as $n$ increases. Resolving this issue, e.g. through ensuring a polynomial-size grid, remains an open problem. Another open problem is how many arcs we need if we restrict solutions to a polynomial-size grid.

Theorem 5. Every triangulation admits a circular arc drawing that uses at most (5n$11) / 3=5 e / 9-1 / 3$ arcs. This drawing can be computed in $O(n)$ time.

Proof. We first analyze the number of circular arcs in our drawing. We perform our algorithm using the canonical order induced by the canonical ordering tree in the minimal Schnyder realizer having the fewest leaves; without loss of generality, let $T_{n}$ be this tree. Recall that $T_{n}$ has at most $\left(2 n-5-\Delta_{0}\right) / 3$ leaves; since $\Delta_{0} \geq 0$, we simplify this to $(2 n-5) / 3$ for the remainder of the analysis. We start with one circle and subsequently process $v_{n}, \ldots, v_{4}$, adding one circular arc per vertex (representing edges in $T_{1}$ and $T_{2}$ ) and a number of line segments (representing edges in $T_{n}$ ). Note that processing $v_{3}$ has no effect since the edge $v_{1} v_{2}$ is the bottom arc. Counting the circle as one arc, we thus have $n-2$ arcs in total. At every vertex in $T_{n}$, one incoming edge is collinear with the outgoing one towards the root. Hence, we charge each line segment uniquely to a leaf of $T_{n}$ : there are at most $(2 n-5) / 3$ segments.

Thus, the total visual complexity is at most $n-2+(2 n-5) / 3=(5 n-11) / 3$. In particular, this shows that, with circular arcs, we obtain greater expressive power for a nontrivial class of graphs in comparison to the $2 n$ lower bound that is known for drawing triangulations with line segments. Since a triangulation has $e=3 n-6$ edges, the visual complexity can also be expressed as $5 e / 9-1 / 3$.

We complete the proof by considering the time bound. The canonical order can be computed in linear time [9]. We then process the vertices in reverse canonical order. Processing a vertex $v$ of degree $d$ takes $O(1+d)$ time: we need only a constant number of computations to place arc $A$ and find the subarc where we can place the undrawn neighbors of $v$; the placement of these takes $O(1)$ per neighbor. Since the graph is planar, the sum over degrees is linear, and thus we obtain an $O(n)$ algorithm for computing this drawing.

This bound readily improves upon the result for line segments $(7 e / 9-10 / 3)$ by Durocher and Mondal [11]. Schulz [24] proved an upper bound of $2 e / 3$ arcs. The bound above is an improvement on this result, though only for triangulations.

Degrees of freedom. One circular arc has five degrees of freedom (DoF), which is one more than a line segment. Hence, our algorithm with circular arcs uses at most 

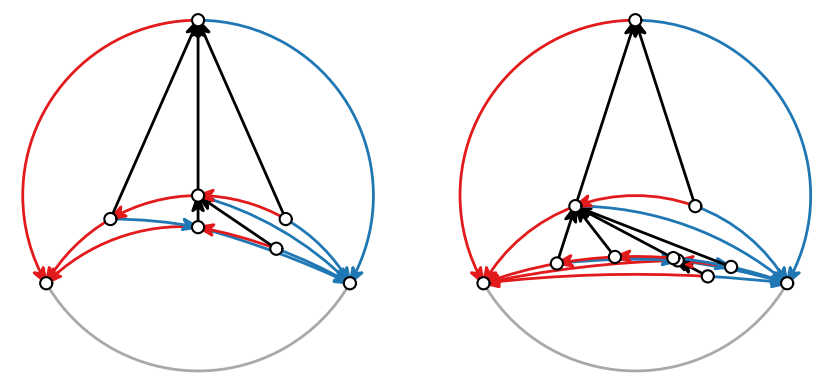

Figure 19: Drawings produced by the algorithm with $n=7$ (left) and $n=10$ (right).

$5 \cdot(5 n-11) / 3=(25 n-55) / 3$ DoF, even if we disregard any DoF reduction arising from the need to have arcs coincide at vertices. This remains an improvement over the result of Durocher and Mondal [11, using at most $4 \cdot(7 n-10) / 3=(28 n-40) / 3$ DoF. The lower bound for line segments $(4 \cdot 2 n=24 n / 3)$ is lower than what we seem to achieve with our algorithm. However, our algorithm uses line segments rather than $\operatorname{arcs}$ to draw the tree $T_{n}$. Thus, the actual DoF employed by the algorithm is $5(n-2)+4 \cdot(2 n-5) / 3=(23 n-50) / 3$, which is in fact below the lower bound for line segments.

4-connected triangulations. We may further follow the rationale of Durocher and Mondal [11] by applying a result by Zhang and He [27]. Using regular edge labelings, they proved that a 4-connected triangulation admits a canonical ordering tree with at most $\lceil(n+1) / 2\rceil$ leaves [27]. Applying this to our analysis, we find that our algorithm uses at most $n-2+\lceil(n+1) / 2\rceil=\lceil(3 n-3) / 2\rceil \leq 3 n / 2-1$ arcs.

Theorem 6. Every 4-connected triangulation admits a circular arc drawing that uses at most $3 n / 2-1=e / 2+2$ arcs. This drawing can be computed in $O(n)$ time.

\section{$6 \quad$ Planar graphs with circular arcs}

The algorithm for triangulations of the previous section easily adapts to draw a general planar graph $G$ with $n \geq 3$ vertices and $e$ edges. As connected components can be drawn independently, we assume $G$ is connected. We need to only triangulate $G$, thereby adding $3 n-e-6$ chords; this takes linear time and can immediately also produce the necessary canonical order (e.g. Section 6 of [16]). We then run the algorithm described in Theorem 5 . Finally, we remove the chords from the drawing. Each chord may split an arc into two arcs, thereby increasing the total complexity by one. From Theorem 5 , it follows that we obtain a drawing of $G$ using $(5 n / 3-11 / 3)+(3 n-e-6)=14 n / 3-e-29 / 3$ arcs. Since $e$ is at least $n-1$, a very rough upper bound is $11 n / 3-26 / 3$.

Theorem 7. Every planar graph with $n \geq 3$ admits a circular arc drawing with at most $14 n / 3-e-29 / 3$ arcs. This drawing can be computed in $O(n)$ time.

Again, this bound readily improves upon the upper bound for line segments $(16 n / 3-$ $e-28 / 3$ ) by Durocher and Mondal [11. Provided the graph is 3-connected, Schulz's [24] bound of $2 e / 3-1$ is lower than our bound, but only for sparse-enough graphs having $e<14 n / 5-26 / 5$. However, there are planar graphs that are not 3 -connected with as many as $3 n-7$ edges (one less than a triangulation): there is no sparsity for which planar graphs must be 3-connected and Schulz's bound is lower than our result. In case the original graph $G$ is 4-connected, extending it to a triangulation by adding edges does not violate this property. Repeating the above analysis using the improved bound of Theorem 6 yields us the following result. 
Theorem 8. Every 4-connected planar graph admits a circular arc drawing with at most $9 n / 2-e-7$ arcs. This drawing can be computed in $O(n)$ time.

Since any planar graph can be drawn trivially with $e$ arcs (or line segments), the above results are an improvement over the trivial bound, only if $e>14 n / 3-e-29 / 3$ for planar graphs or $e>9 n / 2-e-7$ for 4 -connected planar graphs. That is, we need $e>7 n / 3-29 / 6$ or $e>9 n / 4-7 / 2$, fairly dense graphs.

Heuristic improvement. We investigate here a simple improvement upon the above by choosing a "good triangulation". However, we must finally conclude that for worst-case bounds, this improvement is not noticeable.

For the improvement, we observe that at most two pairs of edges at every vertex share an arc: two edges on the horizon and two edges of $T_{n}$. We thus reduce the necessary geometric primitives by choosing a specific triangulation: for every face $\left\langle v_{1}, \ldots, v_{k}, v_{1}\right\rangle$ with $k>3$, we pick a single vertex, say $v_{1}$, and triangulate the face by connecting $v_{1}$ to $v_{3}, \ldots, v_{k-1}$ with temporary chords. Note that planarity ensures that there is a vertex for every face such that we can add these edges without creating multi-edges: the graph induced by $v_{1}, \ldots, v_{k}$ is outerplanar, so it has a degree- 2 vertex. This way, when we remove the temporary chords in the final step of the algorithm, the number of arcs that are split into two can be reduced.

We may even further save on complexity by selecting the same vertex for multiple adjacent faces. Bose et al. 2] have shown that one can select in $O(n)$ time a set $S$ of at most $\lfloor n / 2\rfloor$ vertices such that every face is incident to at least one vertex of this set. Note that we would need to only cover faces of size $|f| \geq 4$, as triangular faces do not receive any temporary chords. We now triangulate each face to one of its incident vertices in $S$. This means that the temporary chords do not increase the visual complexity, except for up to two chords at every vertex in $S$.

We define the total reduction $R$ as the number of arcs that no longer cause a split of an arc in the analysis of general planar graphs. The complexity bound thus becomes $14 n / 3-e-29 / 3-R$. By the above rationale, we can express $R$ as

$$
\sum_{f \in F}(|f|-3)-2|S|=\sum_{f \in F}|f|-3|F|-2|S|=2 e-3|F|-2 S,
$$

where $F$ is the set of faces in the graph $G$. By Euler's formula, we have $|F|=2+e-n$ and we know $S \leq n / 2$ by the result of Bose et al. [2]. We this get that $R \geq 2 n-e-6$.

We can thus guarantee that this heuristic provides a reduction if $e<2 n-6$, that is, for sparse enough graphs. Under this assumption, we find that the final bound on the number of arcs is

$$
\frac{14 n}{3}-e-\frac{29}{3}-R=\frac{14 n}{3}-e-\frac{29}{3}-2 n+e+6=\frac{8 n}{3}-\frac{11}{3} .
$$

It is clear that the trivial bound of $e$ performs better than this heuristic for rather sparse graphs, that is, when $e>8 n / 3-11 / 3$. As we need $e<2 n-6$ for a the worst-case reduction to be positive, we thus need that $8 n / 3-11 / 3<e<2 n-6$. This is however not possible for any positive $n$.

We must conclude that this improvement has no effect in the worst-case scenario on the complexity bound for general planar graphs. This is because the need for large faces for the improvement to be noticeable is directly opposite the need for a dense graph for our bound to be better than the trivial bound. However, this method can still be used as a heuristic and does reduce visual complexity, when a graph has multiple faces of size at least 6 , or when we can find a suitable small set $S$. 
Improvements on $S$ to cover the non-triangular faces would readily improve the bounds here as well. For example, we may also take the trivial bound $|S| \leq|F|$. However, going through the same analysis tells us that the reduction must be positive if $e<5 n / 8-10 / 8$, that is, for even sparser graphs - the bound by Bose et al. [2].

\section{Conclusions}

We investigated the visual complexity of graphs: the number of line segments or circular arcs needed to draw a planar graph.

We provided algorithms to construct segment drawings for various planar graph classes, such that the resulting coordinates are bounded integers; in other words, we bound the height and width of the integer grid needed for the drawing. In particular, we have shown how to draw trees of $n$ vertices on an $O\left(n^{2}\right) \times O\left(n^{1.58}\right)$ grid using at most $3 n / 4$ segments. This algorithm can be adapted to achieve the optimal $\theta / 2$ segments, where $\theta$ is the number of odd degree vertices, if we increase the grid size to be quasi-polynomial. Moreover, we described an algorithm to draw maximal outerplanar graphs and planar 3-trees on an $O(n) \times O\left(n^{2}\right)$ grid, using at most $3 n / 2$ and $(8 n-17) / 3$ segments respectively.

Finally, we provided a linear-time algorithm to construct a drawing of an $n$-vertex triangulation using at most $(5 n-11) / 3$ arcs, though coordinates are not restricted to an integer grid. This readily gives an algorithm for general planar graphs as well; for both cases, having a 4-connected graph reduces the number of arcs needed.

Open problems. There remain a number of interesting avenues for further research. Can we draw trees using $\theta / 2$ segments on a polynomially-sized grid? For arc drawings, no results on bounded grids are known beyond the result for trees by Schulz [24]. In general, stronger lower bounds beyond the general counting arguments (see introduction) are missing; this prevents us from arguing optimality for many cases, beyond those where the lower bounds can actually always be achieved.

\section{References}

[1] N. Bonichon, B. L. Saëc, and M. Mosbah. Wagner's theorem on realizers. In P. Widmayer, F. T. Ruiz, R. M. Bueno, M. Hennessy, S. Eidenbenz, and R. Conejo, editors, Proc. 29th Int. Coll. Automata, Languages and Programming (ICALP'02), volume 2380 of Lecture Notes Comput. Sci., pages 1043-1053. Springer, 2002. doi: $10.1007 / 3-540-45465-9 \_89$.

[2] P. Bose, D. Kirkpatrick, and Z. Li. Worst-case-optimal algorithms for guarding planar graphs and polyhedral surfaces. Comput. Geom., 26(3):209-219, 2003. doi:10.1016/ s0925-7721(03)00027-0.

[3] E. Brehm. 3-orientations and Schnyder 3-tree-decompositions. Master's thesis, Freie Universität Berlin, 2000. URL: http://page.math.tu-berlin.de/ felsner/ Diplomarbeiten/brehm.ps.gz.

[4] S. Chaplick, K. Fleszar, F. Lipp, A. Ravsky, O. Verbitsky, and A. Wolff. Drawing graphs on few lines and few planes. In Y. Hu and M. Nöllenburg, editors, Proc. 24th Int. Symp. Graph Drawing Netw. Vis. (GD'16), volume 9801 of Lecture Notes Comput. Sci., pages 166-180. Springer, 2016. doi:10.1007/978-3-319-50106-2_14. 
[5] S. Chaplick, K. Fleszar, F. Lipp, A. Ravsky, O. Verbitsky, and A. Wolff. The complexity of drawing graphs on few lines and few planes. In F. Ellen, A. Kolokolova, and J. Sack, editors, Proc. 15th Int. Symp. Algorithms Data Struct. (WADS'17), volume 10389 of Lecture Notes Comput. Sci., pages 265-276. Springer, 2017. doi: 10.1007/978-3-319-62127-2_23.

[6] M. Chrobak and T. H. Payne. A linear-time algorithm for drawing a planar graph on a grid. Inform. Process. Lett., 54:241-246, 1995.

[7] H. de Fraysseix and P. O. de Mendez. On topological aspects of orientations. Discrete Math., 229(1-3):57-72, 2001. doi:10.1016/S0012-365X(00)00201-6.

[8] H. de Fraysseix, J. Pach, and R. Pollack. Small sets supporting fary embeddings of planar graphs. In J. Simon, editor, Proc. 20th Ann. ACM Symp. Theory Comput. (STOC'88), pages 426-433. ACM, 1988. doi:10.1145/62212.62254.

[9] H. de Fraysseix, J. Pach, and R. Pollack. How to draw a planar graph on a grid. Combinatorica, 10(1):41-51, 1990. doi:10.1007/BF02122694.

[10] V. Dujmović, D. Eppstein, M. Suderman, and D. R. Wood. Drawings of planar graphs with few slopes and segments. Comput. Geom. Theory Appl., 38(3):194-212, 2007. doi:10.1016/j.comgeo.2006.09.002.

[11] S. Durocher and D. Mondal. Drawing plane triangulations with few segments. In M. He and N. Zeh, editors, Proc. 26th Canad. Conf. Comput. Geom. (CCCG'14), pages 40-45. Carleton Univ., 2014. URL: http://www.cccg.ca/proceedings/2014/ papers/paper06.pdf.

[12] S. Durocher, D. Mondal, R. I. Nishat, and S. Whitesides. A note on minimumsegment drawings of planar graphs. J. Graph Algorithms Appl., 17(3):301-328, 2013. doi:10.7155/jgaa.00295.

[13] S. Felsner and W. T. Trotter. Posets and planar graphs. J. Graph Theory, 49(4):273284, 2005. doi:10.1002/jgt.20081.

[14] G. Hültenschmidt, P. Kindermann, W. Meulemans, and A. Schulz. Drawing planar graphs with few geometric primitives. In H. L. Bodlaender and G. J. Woeginger, editors, Proc. 43rd Int. Workshop Graph-Theor. Concepts Comput. Sci. (WG'17), volume 10520 of Lecture Notes Comput. Sci., pages 316-329. Springer, 2017. doi: 10.1007/978-3-319-68705-6_24.

[15] A. Igamberdiev, W. Meulemans, and A. Schulz. Drawing planar cubic 3-connected graphs with few segments: Algorithms and experiments. In E. Di Giacomo and A. Lubiw, editors, Proc. 23rd Int. Symp. Graph Drawing Netw. Vis. (GD'15), volume 9411 of Lecture Notes Comput. Sci., pages 113-124. Springer, 2015. doi:10.1007/ 978-3-319-27261-0_10.

[16] G. Kant. Algorithms for Drawing Planar Graphs. PhD thesis, Universiteit Utrecht, 1993.

[17] P. Kindermann, W. Meulemans, and A. Schulz. Experimental analysis of the accessibility of drawings with few segments. In F. Frati and K.-L. Ma, editors, Proc. 25th Int. Symp. Graph Drawing Netw. Vis. (GD'17), volume 10692 of Lecture Notes Comput. Sci., pages 52-64. Springer, 2017. doi:10.1007/978-3-319-73915-1_5. 
[18] M. Kryven, A. Ravsky, and A. Wolff. Drawing graphs on few circles and few spheres. In B. S. Panda and P. P. Goswami, editors, Proc. 4th Int. Conf. Algorithms Discrete Appl. Math. (CALDAM'18), volume 10743 of Lecture Notes in Computer Science, pages 164-178. Springer, 2018. URL: https://doi.org/10.1007/ 978-3-319-74180-2_14, doi:10.1007/978-3-319-74180-2_14.

[19] D. R. Lick and A. T. White. k-degenerate graphs. Canad. J. Math., 22:1082-1096, 1970. doi:10.4153/CJM-1970-125-1

[20] D. Mondal. Visualizing graphs: optimization and trade-offs. phdthesis, University of Manitoba, 2016. URL: http://hdl.handle.net/1993/31673.

[21] D. Mondal, R. I. Nishat, S. Biswas, and M. S. Rahman. Minimum-segment convex drawings of 3-connected cubic plane graphs. J. Comb. Optim., 25(3):460-480, 2013. doi:10.1007/s10878-011-9390-6.

[22] D. J. Rose. On simple characterizations of k-trees. Discrete Math., 7(3):317-322, 1974. doi:10.1016/0012-365X(74)90042-9.

[23] W. Schnyder. Embedding planar graphs on the grid. In D. S. Johnson, editor, Proc. 1st Ann. ACM-SIAM Symp. Discrete Algorithms (SODA'90), pages 138-148. SIAM, 1990. URL: http://dl.acm.org/citation.cfm?id=320191.

[24] A. Schulz. Drawing graphs with few arcs. J. Graph Algorithms Appl., 19(1):393-412, 2015. doi:10.7155/jgaa.00366.

[25] R. E. Tarjan. Data Structures and Network Algorithms, chapter 5 - Linking and Cutting Trees, pages 59-70. SIAM, 1983. doi:10.1137/1.9781611970265.ch5.

[26] G. A. Wade and J. Chu. Drawability of complete graphs using a minimal slope set. Comput. J., 37(2):139-142, 1994. doi:10.1093/comjnl/37.2.139.

[27] H. Zhang and X. He. Canonical ordering trees and their applications in graph drawing. Discrete Comput. Geom., 33(2):321-344, 2005. doi:10.1007/s00454-004-1154-y. 